\title{
Application of Shadow Matching Technique to Improve Smartphone-based Global Navigation Satellite System Positioning Accuracy
}

\author{
Dokyun Kim, ${ }^{1}$ Mingyun Jang, ${ }^{2}$ Kirim Lee, ${ }^{3}$ and Wonhee Lee ${ }^{2 *}$ \\ ${ }^{1}$ Department of Civil Engineering, Gyeongbuk Provincial University, Yecheon 36830, Korea \\ ${ }^{2}$ School of Convergence and Fusion System Engineering, Kyungpook National University, Sangju 37224, Korea \\ ${ }^{3}$ Department of Spatial Information, Kyungpook National University, Daegu 41566, Korea
}

(Received October 26, 2021; accepted January 4, 2022)

Keywords: GNSS, shadow matching, 3D surface model, GIS, sky mask method, skyline analysis, barrier analysis

In this study, the smartphone global navigation satellite system (GNSS) positioning accuracy was improved by selecting optimal visible satellites through a 3D surface model and the shadow matching (SM) technique. A 3D surface model was constructed using an unmanned aerial vehicle (UAV) to obtain an accurate terrain model and perform visibility analysis. Additionally, we used the geographic information system (GIS) analysis as well as the skyline and barrier analysis methods to calculate the visibility between smartphones and satellites. The altitudes of the satellites were calculated to analyze the visibility between the analyzed smartphone and the satellites, and the visible satellites were selected by a sky mask method. Visible satellites were classified through the analysis of the signal characteristics by investigating the observed elevation angle of the satellite signal, the carrier-to-noise ratio $(\mathrm{C} / \mathrm{No})$, and the pseudorange ratio consistency (Prc). Moreover, the satellites were categorized via two classification methods and then recombined by statistical analysis to optimally select the visible satellites. Furthermore, the smartphone's location was computed using the optimal combination of satellites, and the accuracy was evaluated by comparing the calculated location coordinates with the true position coordinates. As a result, the maximum rates of improvement were 880,356 , and $5 \%$ in environments of low-rise building urban, high-rise building urban, and surrounded by tall buildings, respectively.

\section{Introduction}

Network technology is rapidly developing, and smartphones now provide comprehensive cultural services beyond basic communication, such as information retrieval, transportation, and finance. ${ }^{(1)}$ Smartphones transcend the limitations of time and space in terms of network connectivity, allowing users to freely use the information or services they need anytime and anywhere without restrictions. As the number of smartphone users increases, location-based

*Corresponding author: e-mail: wlee33@,knu.ac.kr

https://doi.org/10.18494/SAM3714 
services (LBSs) are rapidly becoming widespread, and accurate location information for user convenience is an essential task. ${ }^{(2)}$ LBSs provide various additional services by identifying the location of a moving person with a mobile phone or terminal. The satellite navigation chipset in a smartphone allows users to check their location and provides real-time geographic information about the individual via the mobile environment.

A network-signal-based positioning method collects location information using a communication network's base station reception signal; a Bluetooth-based method uses a Bluetooth beacon, and a wireless local area network method utilizes a Wi-Fi wireless router. Additionally, a ubiquitous positioning method employs a ubiquitous computing device together with a satellite-signal-based positioning method that uses satellite signals, such as the global positioning system (GPS), and a mixed positioning method in which these methods are mixed. ${ }^{(3-6)}$

Positioning technology using satellite signals is advantageous over other positioning technologies since it has greater accuracy and does not require a separate device. However, lowcost GNSS receiving chips installed in mobile terminals generate errors of tens to hundreds of meters. The reliability of the location information decreases in areas where GNSS satellite signals are blocked by high-rise buildings, trees, and other structures, and/or in the case of multipath propagation where satellite signals are received through multiple paths. ${ }^{(7)}$ The problem caused by the insufficient number of visible satellites in environments surrounded by high buildings is solved by integrating other satellite navigation systems with GNSS.

In May 2016, Google announced that it would provide raw data of the global navigation satellite system (GNSS) in smart devices supporting the Android Nougat ver. 7 operating system. ${ }^{(8)}$ When GNSS raw data is used, users can proactively improve and diversify the accuracy of the LBS. Currently, many open-source applications or programs using a large amount of GNSS information are being developed for smartphone location services, and research is being conducted to improve the location accuracy of smartphones.

Groves confirmed that it is difficult to distinguish between non-line-of-sight (NLOS) and line-of-sight (LOS) signals using the signal-noise ratio (SNR) measurement due to the properties of smartphone antennas since the SNR measurement process can also be considered to introduce noise. ${ }^{(9)}$ Ercek et al. showed that an accurate positioning solution cannot be determined without obtaining the LOS from four or more satellites. They suggested that a degraded location solution can be obtained using signal reflection via surrounding buildings or vehicles. ${ }^{(10)}$ Groves showed, via signal geometry calculations in the satellite signal analysis of the study area, that the location accuracy is higher in the direction of traffic along a road than when crossing the road. ${ }^{(11)}$ Wang et al. showed that the shadow matching (SM) technique can discriminate the direction of whether the user is crossing the road or traveling in the direction of traffic in the surrounding environment, and that it can accurately identify a surrounding surface with GPS and GNSS measurements with a geodetic receiver. ${ }^{(12)}$ Wang et al. confirmed that the elevation of a building boundary can be calculated in the range of the azimuth. ${ }^{(13)}$ Wang et al. described a new positioning technique that determines location by comparing the signal availability and signal strength measured by the GNSS SM technique with predictions made using a 3D surface model. They showed that when the measured GPS data and Russian global navigation satellite system 
(GLONASS) data are used, the SM technique has significantly higher performance than the existing GNSS positioning in the horizontal direction. ${ }^{(14)}$

The SM method classifies the visible satellite signals among the observed satellite signals and compares the visible satellites predicted by the sky mask method using the ephemeris and the visible range. Visibility is analyzed by calculating the relative location of the 3D surface model that reflects the surrounding trees and other structures, yielding a realistic analysis.

The aim of this study is to build a 3D surface model using unmanned aerial vehicles (UAVs) to improve the accuracy of smartphone satellite navigation measurements in areas with high shielding properties or in urban areas with high multipath propagation using SM algorithms and GIS analysis techniques.

\section{Materials and Methods}

\subsection{Research method}

In this study, the SM technique was used to improve the accuracy of the smartphone GNSS. The principle of this technique is to analyze the visibility of satellites using the values of the observed satellite signal characteristics to compare their visibility predicted by the sky mask method. The SM technique is a positioning technology using a 3D surface model. For the visibility analysis at the smartphone's location, a 3D surface model was built using a UAV, and the satellites' locations were calculated from the ephemeris (Fig. 1).

The visible range was calculated using the skyline and barrier analysis methods as well as the ArcGIS analysis method to analyze the visibility between the smartphone and the satellites. By calculating the visible range and the satellites' altitudes, the visible satellites were selected by the sky mask method, and the values of the observed satellite signal characteristics were analyzed to

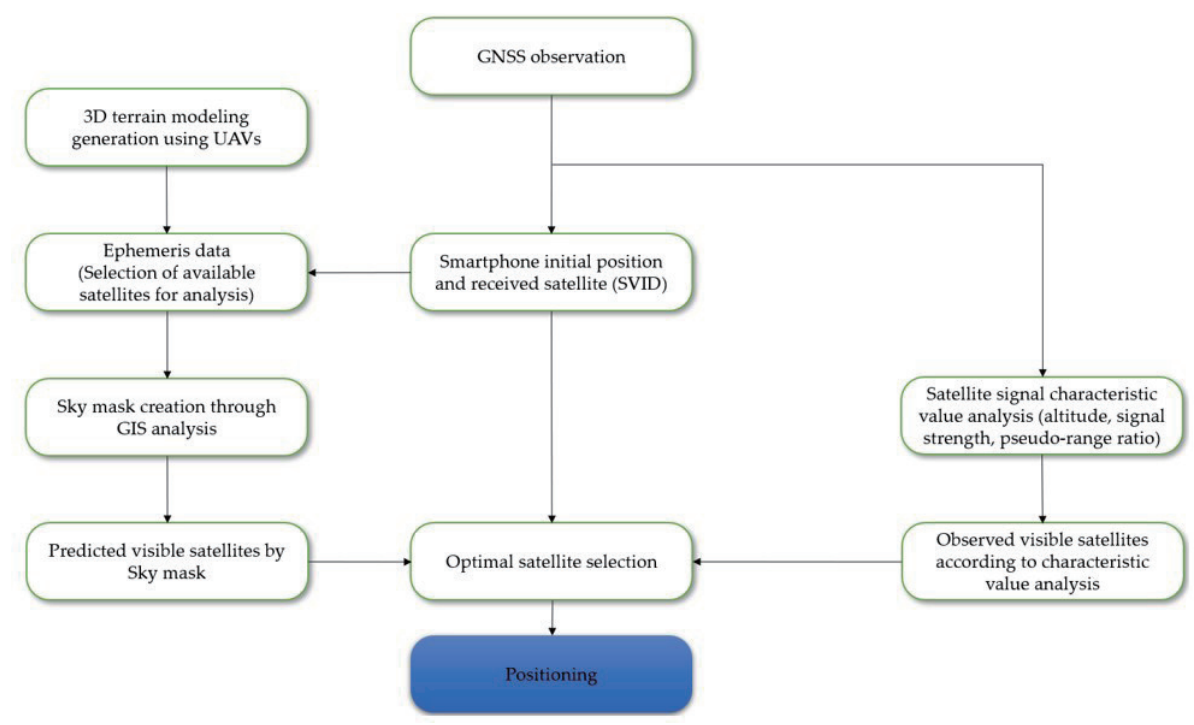

Fig. 1. (Color online) Research flow chart. 
classify the visible satellites through the analysis of the signal characteristics. The best visible satellite combination was calculated by combining satellites classified using two classification methods: sky mask method and observation satellite signal characteristic value analysis. By calculating coordinates using the combined satellites and comparing them with the true position coordinates, we present the possibility of applying the SM technique to improve GNSS accuracy for smartphones.

\subsection{Shadow matching method}

High-rise buildings, trees, and other structures in a city center can block and reflect satellite signals, making GNSS location information unreliable. The SM technique is a method of improving the accuracy of urban location information by using a 3D surface model and GNSS information. The SM technique determines a location by comparing the received signal visibility with the predicted signal visibility. The classification of the LOS and NLOS signals of GNSS is a critical factor in the SM technique and existing positioning methods.

The existing SM technique was proposed by Groves ${ }^{(11)}$ and has since been improved by Groves and coworkers. ${ }^{(15-17)}$ It calculates a predicted satellite signal using 3D building information with multiple points as candidate points and then determines the user's location with greater accuracy by comparing it with the observed satellite signal. On the basis of the detailed concept in Fig. 2, the user can expect that the direct signal received from the satellite or the location where the visible signal is received can be located, and the signal is shielded by the building. The user is not located when an invisible signal is received. ${ }^{(18,19)}$

As shown in Fig. 3(a), the SM method selects a search area based on the 3D building database and the receiver's initial position and predicts the visible satellites at each point by classifying the candidate points in a grid form for visibility analysis. The position of each candidate point is given a score by comparing the predicted visible satellite at each point with the satellite observed at the initial position. As shown in Fig. 3(b), the candidate point with the highest score is displayed in red.
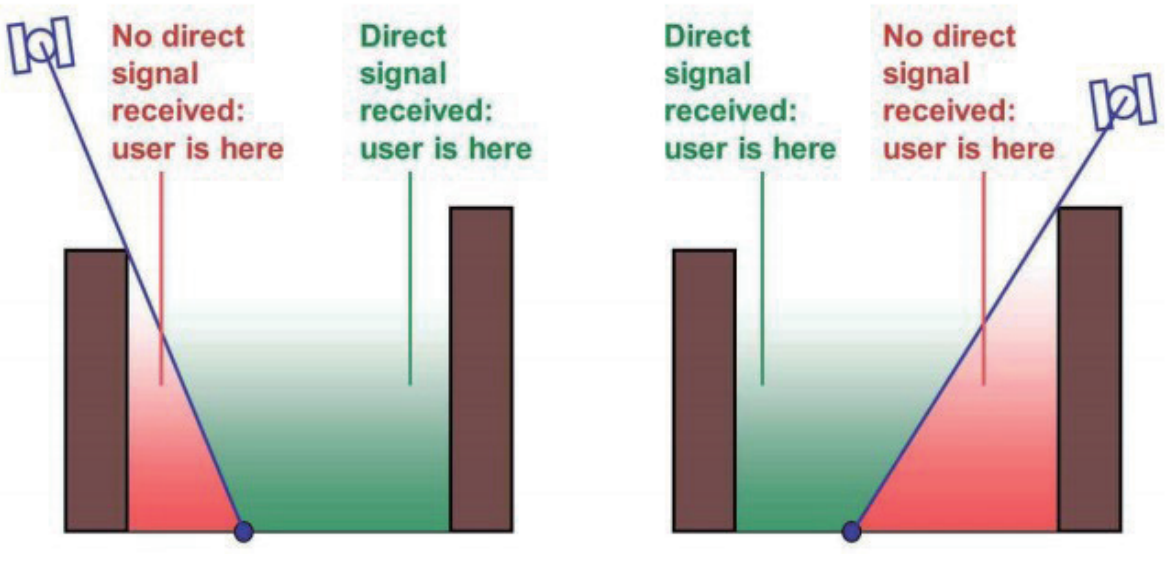

Fig. 2. (Color online) Principle of SM technique..$^{(11)}$ 


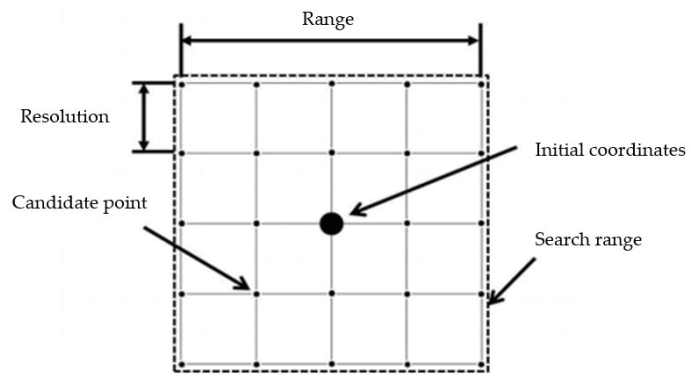

(a)

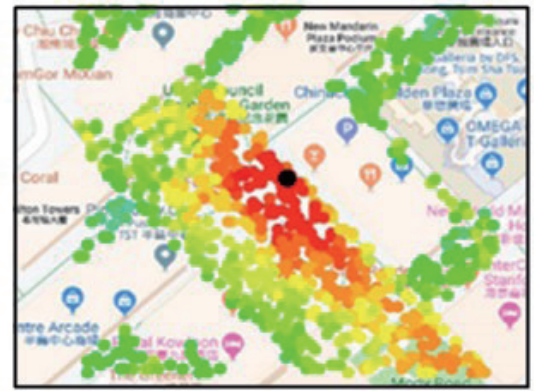

(b)

Fig. 3. (Color online) Technique: (a) grid point configuration diagram ${ }^{(20)}$ and (b) candidate point classification (score) map. ${ }^{(21)}$

The candidate point having the highest score is highly likely to be the true position. There may be multiple candidate points with high scores; in this case, the final location is calculated by averaging the coordinates of the candidate points.

Methods using 3D surface models and the SM technique are being studied extensively for use in urban areas. However, these methods can lead to incorrect results because the grid size of the candidate points has a significant effect on the positioning accuracy, and they predict the same satellite in a building area with a similar geometry according to the initial position setting of the receiver. Since the smartphone used in this study does not have a high reliability of the initial position and receives many satellite signals through multipath propagation, the previous research methods cannot provide good results. Therefore, in this study, the visible satellites were predicted using the 3D surface model and the sky mask method by the existing SM technique; the visible satellites were selected using the values of the receiving satellite signal characteristics. Then, by analyzing the signals received from the satellites, we improved the positioning accuracy of the smartphone.

\subsubsection{Predicted visible satellites}

In urban areas where buildings are dense, low-altitude satellite signals are blocked by nearby tall buildings. High-angle satellites directly transmit satellite signals to the receiver, whereas low-angle satellites are shielded by buildings or delivered via multipath propagation. This phenomenon is severe in urban areas, especially when there are many tall buildings around. The multipath error causes large errors when determining the user's location using satellite navigation. Therefore, it is necessary to accurately classify the 3D surface model and multipath signal for satellite visibility analysis.

\subsubsection{Observed visible satellites}

It would be convenient to use all satellite signals observed in GNSS for the visibility analysis of the observed satellite signals, but the observed satellite signals include both visible and invisible satellite signals. The visible satellite signals must be classified by analyzing the signal characteristics of the visible satellite using the the carrier-to-noise ratio $(\mathrm{C} / \mathrm{No})$ as the signal 
strength, the altitude, and the pseudorange ratio consistency (Prc). Generally, the higher the signal strength or altitude, the more likely the signal is from a visible satellite. A high signal strength means that the signal is transmitted directly to the receiver without other reflectors or multipath propagation, and a high-altitude satellite is less likely to be shielded by buildings. When Prc is constant, the signal is unaffected by reflection, multipath propagation, or object diffraction.

\subsubsection{Optimal satellite combination}

Among the satellites classified as visible satellites by the sky mask method, those classified as observed satellites by the analysis of the signal characteristcs are selected as visible satellites (Table 1). Satellites classified as invisible satellites by the sky mask method but classified as visible satellites by the analysis of the signal characteristics are reclassified into visible and invisible satellites after the signal characteristics are analyzed for the satellites selected by the two classification methods. Consequently, there is a possibility that the visible satellites are classified as invisible owing to the uncertainty of the sky mask method resulting from the initial position error of the smartphone. Additionally, satellites classified as visible satellites by the sky mask method but unobserved in the analysis of the signal characteristics are excluded. This classification corrects the incorrect selection error of the visible satellites due to the smartphone's initial position error. The unpredicted and unobserved satellites are excluded from the coordinate calculation. Coordinate calculations are performed using the finally selected satellites.

\subsection{D surface model generation using UAVs}

A 3D surface model is generated to determine whether the signal from a satellite is blocked. Therefore, we compared the elevation angle (EA) of the satellite with the boundary of the modeled buildings and other structures to estimate the multipath error due to reflection and diffraction.

To improve the location accuracy of smartphones in high-rise buildings or narrow alleys, the SM technique was introduced to distinguish whether satellite signals are directly received from the observation location by 3D modeling the surrounding structures and terrain. In this study, DJI's Phantom 4 and the structure from motion (SfM)-based 3DF Zephyr S/W were used for the 3D modeling of the experimental area.

The experimental area was divided into a low-rise building urban environment formed of low-rise buildings, trees, and other structures, a high-rise building urban environment formed of

Table 1

Method of combining the classified satellites after analyzing the predicted visible satellites by the sky mask method and the observed visible satellites by characteristic (C/No, EA, and Prc) analysis.

\begin{tabular}{lccc}
\hline & \multicolumn{2}{c}{ Observation (characterization) } \\
\hline \multirow{2}{*}{$\begin{array}{l}\text { Prediction } \\
\text { (Sky mask method) }\end{array}$} & Measured & Not measured \\
\cline { 2 - 4 } & Invisible & $\begin{array}{c}\text { Comparison of common visibile } \\
\text { satellites and signal characteristics }\end{array}$ & $\times$ \\
\cline { 2 - 4 } & Visible & Select from visible satellites & $\times$ \\
\hline
\end{tabular}


high-rise buildings, and an environment surrounded by tall buildings in which poor reception occurs. For UAV photography, an $80 \%$ overlap was employed to improve accuracy, and horizontal and longitudinal shooting were performed in a double grid format with a $70^{\circ}$ inclination (Table 2). Orthophotos and DEMs were generated using UAV images and 3DF Zephyr S/W for each experimental environment.

The reproducibility of 3D modeling is great, and if accurate location information of each point is included, an accurate sky mask method of the observed point can be obtained. To increase the modeling recall and offset the disadvantages of each registration method, the SfMbased registration method and the registration method through the camera position information were used. The SfM method connects the extracted feature points between the adjacent images, estimates the camera's relative posture and direction with respect to the two images using epipolar geometry, and estimates the 3D position of the feature points. When this process is repeated, the estimated camera position, direction, and 3D position are optimized, and a point cloud is formed. ${ }^{(22)}$ Since the high-density point cloud constructed through the SfM method has relative coordinates, the ground reference points acquired by virtual reference station (VRS) surveying are inputted and converted into absolute coordinates. ${ }^{(23)}$

The area was observed using the VRS observation method. Moreover, the environment of low-rise building urban and the environment surrounded by tall buildings were not observed by the VRS method, so the coordinates were obtained by the total station (T/S) observation method.

In an environment of low-rise building urban, eight points of GCP were used; after image registration, an error of approximately 5 to $17 \mathrm{~cm}$ occurred. In an environment of high-rise building urban, nine points of GCP were used; after image registration, an error of approximately 3 to $16 \mathrm{~cm}$ was calculated. Finally, the error in the environment surrounded by tall buildings was found to be approximately $6-15 \mathrm{~cm}$. Since the cell size was analyzed as $0.5 \mathrm{~m} \times 0.5 \mathrm{~m}$ when constructing the 3D surface model in this study, the calculated error is considered sufficient to proceed with this study. For the ground reference point (true value) of the smartphone observation point in each study area, the coordinates of each point were acquired using the VRS and $\mathrm{T} / \mathrm{S}$ observation methods.

Table 2

Flying location and altitude.

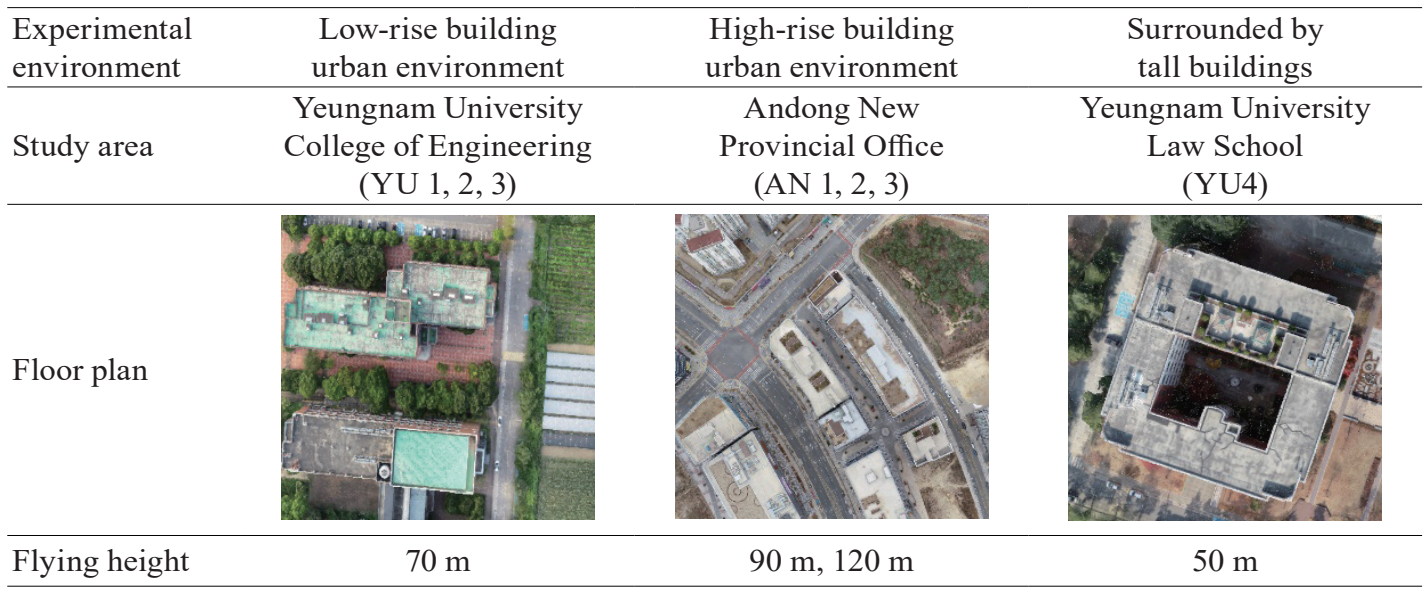




\subsection{Visibility analysis method by sky mask method}

Spatial visibility is greatly affected by the surrounding environment. Basically, the visible range is determined by the influence of buildings, trees, and other structures. When compared to parks and fields, urban areas have limited visibility. In this study, for visibility analysis, the study site was constructed as triangulated irregular network (TIN) data using point clouds of the 3D surface model constructed using UAVs. For spatial analysis, the target site was converted into digital elevation model (DEM) data with a cell size of $0.5 \mathrm{~m} \times 0.5 \mathrm{~m}$. $^{(24)}$ This cell size is assumed appropriate for the spatial analysis when considering the location precision of a smartphone. To calculate the visible range of the smartphone's location, it was analyzed on the basis of the initial location of the smartphone. Generally, when calculating the initial coordinates, an independent positioning method using Prc is used, or an initial position calculated from a navigation device or a smartphone is used. In this study, the initial coordinates of raw data calculated using the smartphone were used. Depending on the surrounding environment, the accuracy of the initial coordinates may be inferior owing to signal reflection, diffraction, or multipath in an area with many signal shielding factors, which may affect the accuracy of the classification of the visible satellites based on the sky mask method. By the SM technique, as in the previous study, the horizontal coordinate used the initial value of the smartphone; the height was analyzed for visibility using that at the horizontal position of the smartphone analyzed in the GIS analysis. Skyline analysis was performed to estimate the visible range using the smartphone's 3D location and DEM data (Fig. 4).

Figure 5(a) is a picture expressing the LOS signal with the satellite from the initial position of the smartphone. When the LOS passes through a building or trees and other structures, the satellite can be determined as an invisible satellite. In Fig. 5(b), satellites corresponding to visible lines above the visible range analyzed by GIS can be determined as visible satellites. For the accuracy of this analysis, a precise 3D surface model and the initial position of the smartphone must be accurate to increase the reliability of the sky mask method.

To calculate the visible range from the location of the smartphone for the visible satellite analysis by the sky mask method and compute the 3D position coordinates of the visible range, the 3D coordinates of the visible range outline are derived through the GIS barrier analysis. The derived coordinates are $3 \mathrm{D}$ coordinates of 365 points at intervals of $1^{\circ}$ from true north. The point positions outside the visible range based on the location of the mobile phone were converted into azimuth and altitude to prepare the sky mask method.

In Fig. 6, when the center corresponds to the smartphone's initial location and the satellite is located in the visible range inside the polygon, it can be determined as a visible satellite. As a result, the outside of the polygon is the invisible range, which can be determined as an invisible satellite. Additionally, most existing visibility analysis studies calculated the visible range using only buildings, but this study analyzed the effects of the buildings, trees, and other structures. In Figs. 4 and 5, the visible range is considerably narrowed by the influence of trees and other structures. Hence, a more realistic analysis is possible by considering the influence of these features. 


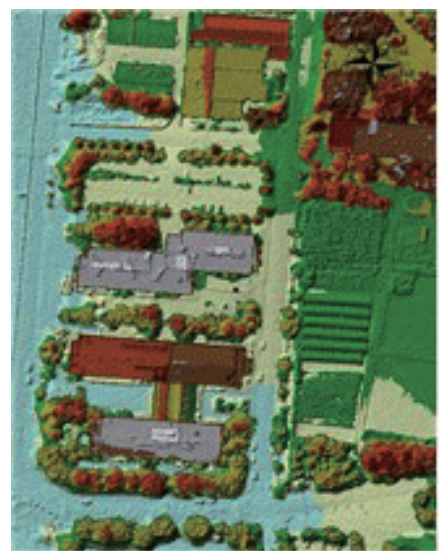

(a)

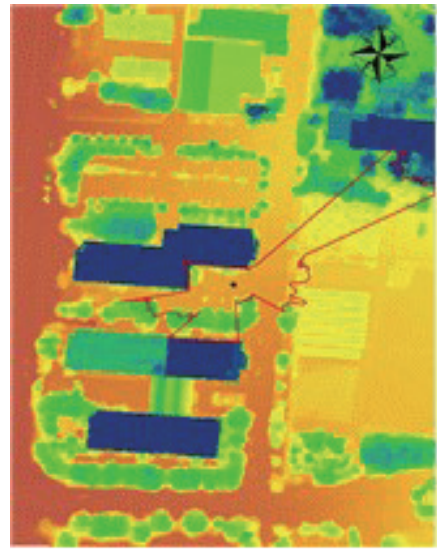

(c)

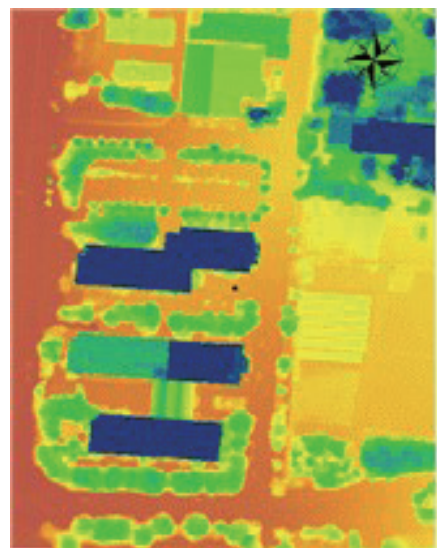

(b)

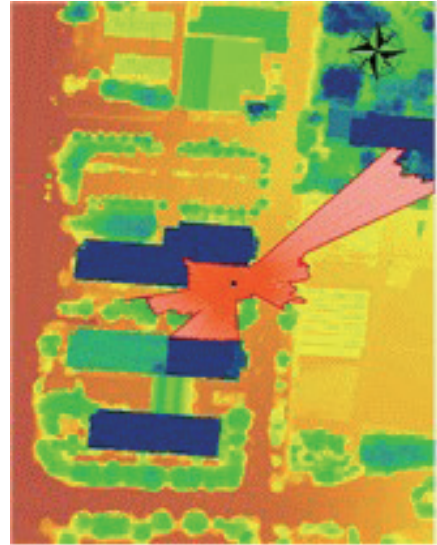

(d)

Fig. 4. (Color online) Examples of skyline and barrier analysis methods are shown to estimate the visible range using the 3D position of the smartphone and DEM data: (a) TIN analysis, (b) DEM analysis, (c) skyline analysis, and (d) barrier analysis.

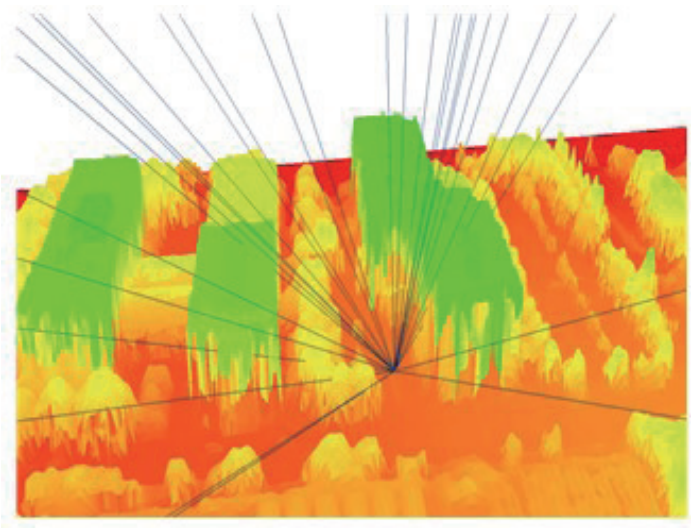

(a)

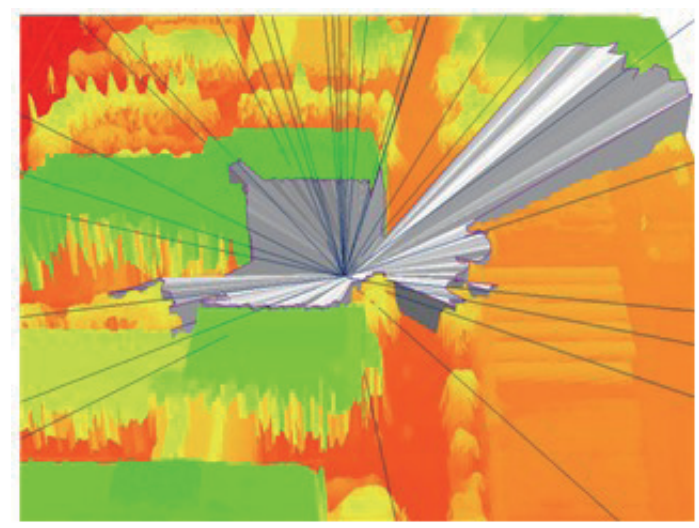

(b)

Fig. 5. (Color online) LOS signal representation of satellites and smartphones: (a) LOS with the satellite from the initial position of the smartphone and (b) satellites corresponding to LOS above the visible range analyzed by GIS. 


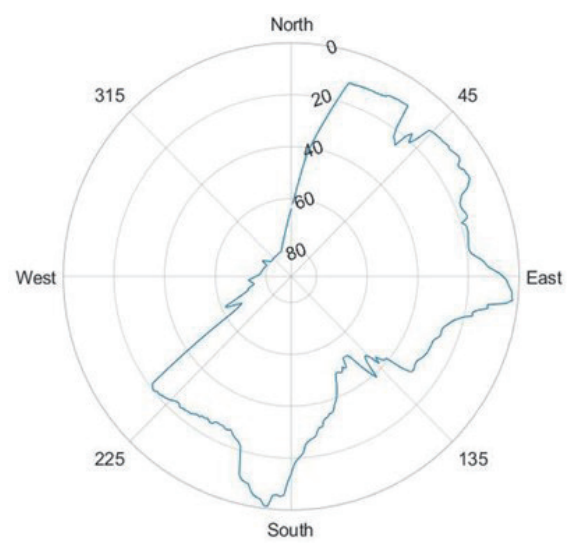

(a)

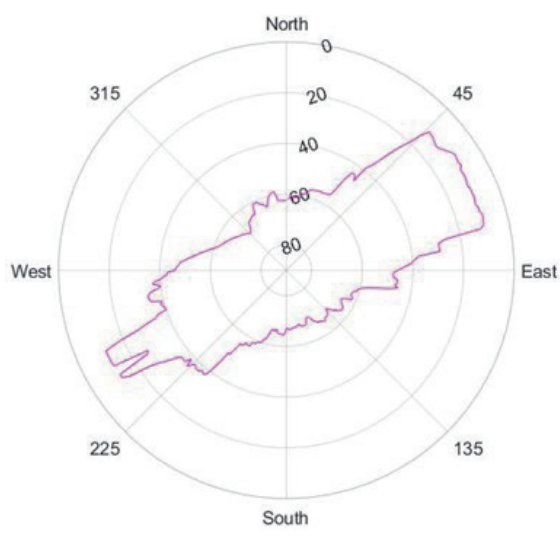

(b)

Fig. 6. (Color online) Example of calculated visible range sky mask method: (a) A2 area and (b) Y3 area.

\subsection{GNSS characterization methods}

Several features are used to distinguish the visibility and invisibility of the satellites based on raw GNSS measurements. Filtering was performed on all receiving satellites using $C / N o$, EA, and Prc. A signal with an EA of less than $15^{\circ}$ among satellite signals received using the minimum mask angle of $15^{\circ}$, which is the standard used in GNSS surveying, is classified as an invisible signal. $^{(25)}$ Next, by calculating the average standard deviation of the Prc and signal strength of all received signals and satellites with a standard deviation or higher, these satellites are classified as invisible satellites. The above method is implemented to classify the visible and invisible satellite signals among the observed satellite signals. Additionally, the average standard deviation is used to adjust the average width of the classification range when considering the smartphone antenna performance. As the classification performance increases, the actual number of available satellites is reduced, affecting the final positioning. Thus, only the signals presenting the difference above the average value among the received signals were classified.

\subsubsection{Elevation angle}

Most satellite signals are more likely to be visible at higher elevations and are unlikely to be blocked by nearby buildings. At the same time, even at a low EA of 30 degrees or less, there is a visibility signal depending on the observation point (Fig. 7). However, most satellites are more likely to be visible at higher EAs.

To calculate the EA of each satellite, it is necessary to estimate the position of each satellite and the approximate position of the user's device. The $i$-th satellite position can be obtained from the precision orbital power data of the reference station as $s^{i}=\left[x^{i} y^{i} z^{i}\right]$ in the Earth-centered, Earth-fixed coordinate system (ECEF) frame. The approximate user location can be calculated using the existing weighted least squares (WLS) localization algorithm or last localization, such as $\hat{p}=[\hat{x} \hat{y} \hat{z}]$. Then, the unit visibility vector from the user's position to the satellite position 


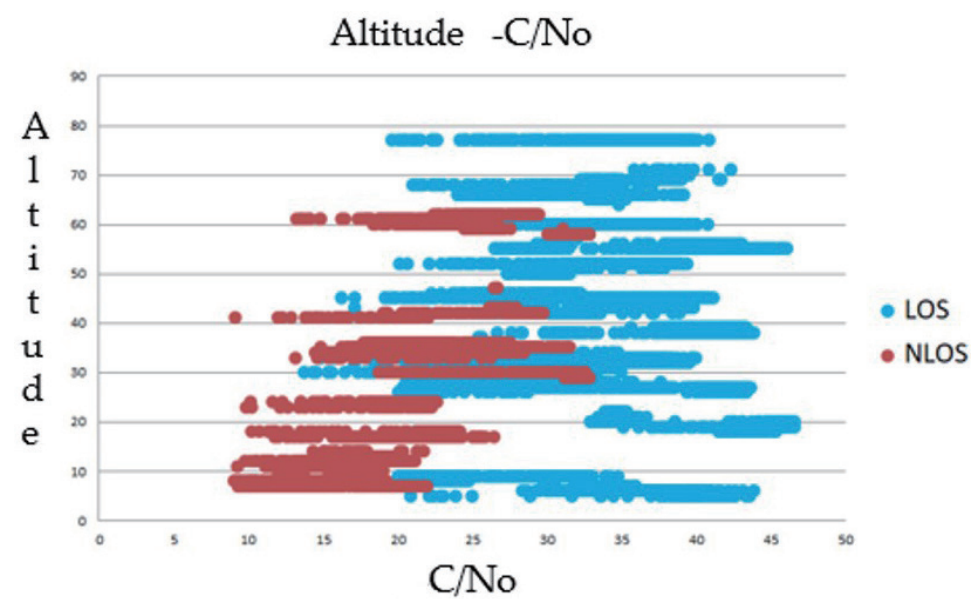

Fig. 7. (Color online) Altitude and C/No.

$\hat{u}=\left[\widehat{u_{x}} \widehat{u_{y}} \widehat{u_{z}}\right]$ is calculated as follows in Eq. (1). The EA of the satellite is calculated using Eq. (2).

$$
\begin{gathered}
\hat{u}=\frac{\left[x^{i}-\hat{x} y^{i}-\hat{y} z^{i}-\hat{z}\right]}{\sqrt{\left(x^{i}-\hat{x}\right)^{2}+\left(y^{i}-\hat{y}\right)^{2}+\left(z^{i}-\hat{z}\right)^{2}}} \\
\hat{\theta}=\arcsin \left(\frac{\left|\widehat{u_{z}}\right|}{\sqrt{+\left(\widehat{u_{x}}+\widehat{u_{y}}\right)^{2}}}\right)
\end{gathered}
$$

\subsubsection{Carrier-to-noise ratio (C/No)}

C/No is generally used to indicate the signal's strength, as shown in Eq. (3).

$$
C / N o=C-(N-B W)
$$

Here, $C$ is the carrier wave intensity, $N$ is the noise intensity, and BW is the noise width. $C / N o$ can be obtained directly from the GNSS raw data. The reflection and diffraction of the satellite signals weaken the signal energy due to energy absorption by building surfaces and other barriers. Therefore, the intensity of the visible signal strength is greater than that of the invisible signal strength. In addition, the signal of the visible satellite shows a uniform distribution without a significant variation in signal strength. The large deviation in satellite signal strength is likely the point at which the signal changes from visible to invisible or where the direction of multipath changes depending on the altitude. By calculating the deviation of the received signal strength, the visibility and invisibility of the satellite signals are classified. ${ }^{(26)}$ 


\subsubsection{Pseudorange ratio consistency (Prc)}

Prc is the rate of change in the pseudorange measurement. This result is calculated by the Doppler transform between the frequencies transmitted by the satellite and received by the receiver for each satellite, and is expressed as ${ }^{(27)}$

$$
P_{R d \cdot i}=-\lambda_{i} \cdot f_{d \cdot i}
$$

Here, $\lambda_{i}$ is the negative value of the carrier wavelength and $f_{d \cdot i}$ is the Doppler shift measurement for satellite $i$. Conversely, the Prc is calculated with Eq. (5), utilizing the user's speed and the satellite in an ideal situation.

$$
P_{R v \cdot i}=\frac{\Delta p_{i}}{\Delta t}=-\left(v_{u s e r}-v_{\text {sat }}^{i}\right) \cdot a^{i}
$$

Here, $P$ is the pseudorange measurement, $\Delta t$ is the time interval between $v_{\text {user }}$ and $v_{\text {sat }}^{i}$ that correspond to the velocity vectors of the user and satellite $i$, respectively, and $a^{i}$ is the unit vector from the user to satellite $i$. In the case of an invisible satellite, the satellite is affected by both reflection and deflection. Thus, the invisible satellite has an unstable pseudorange to Prc compared with that of a visible satellite. The Prc between the two derived values is considered as a characteristic of the satellite signal. Moreover, the $\operatorname{Prc} P_{r c \cdot i}$ at the $t$-th epoch is calculated using the difference as

$$
P_{r c \cdot i}^{t}=P_{R d \cdot i}^{t}-P_{R v \cdot i}^{t}
$$

Visibility and invisibility satellite signal characteristics are determined using Prc.(27) Although the Prc of visible satellites is uniformly distributed, the error range of the Prc of invisible satellites is relatively extensive. In this phenomenon, the path along which the satellite signal moves to the receiver is not constant, and since it is received through several paths, the error width is different from the signal characteristics of the visible satellites (Fig. 8).

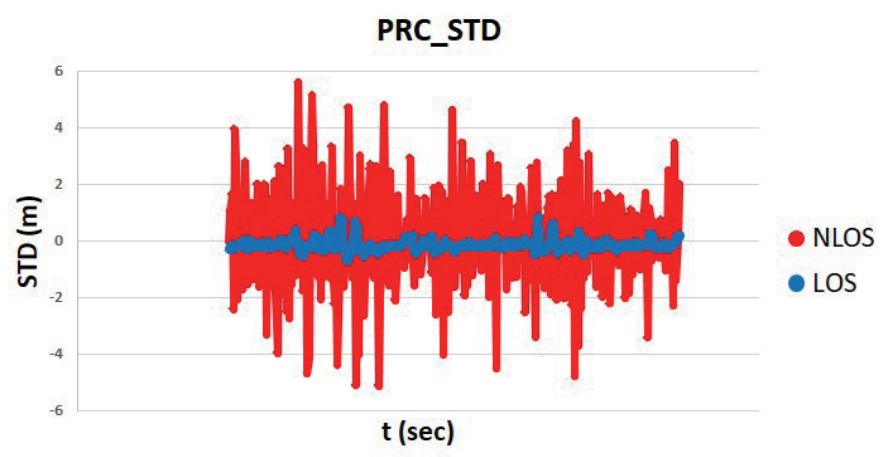

Fig. 8. (Color online) Standard deviation values of the Pre of the visible and invisible satellites. 


\subsubsection{Analysis of GNSS signal characteristics}

To analyze the visibility of the observed satellites by classifying the signal characteristics of the GNSS raw data, standard deviation values of the EA, signal strength, and C/No are calculated, and filtering is performed on all receiving satellites.

The process is noted in the following. First, a signal with an EA of less than $15^{\circ}$ among the satellite signals received using the minimum mask angle of $15^{\circ}$, which is the standard used in GNSS surveying, is classified as an invisible signal. Second, by calculating the average standard deviation of the Prc and signal strength of all receiving satellites, satellites with a standard deviation or higher are classified as invisible satellites.

The above method is implemented to classify the visible and invisible satellite signals among the observed satellite signals, and the average standard deviation is used to adjust the average width of the classification range when considering the smartphone antenna performance. When the classification performance is increased, the actual number of available satellites is reduced, which affects the final positioning, so only the signals showing the difference above the average value among the received signals were classified. Figures 9 and 10 demonstrate the signal characteristics of the visible and invisible satellites.
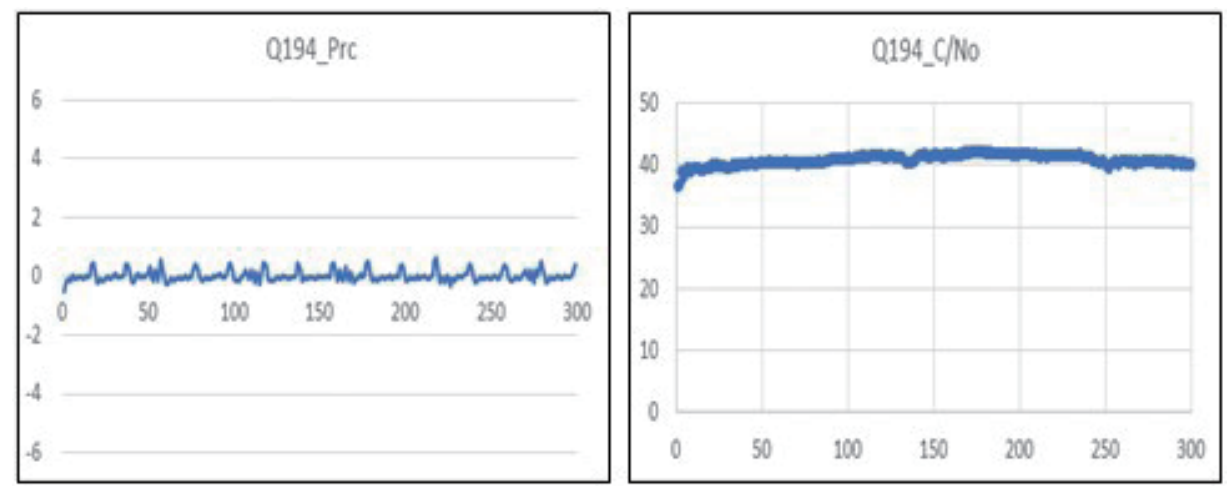

Fig. 9. (Color online) Signal characteristics of the visible satellites.
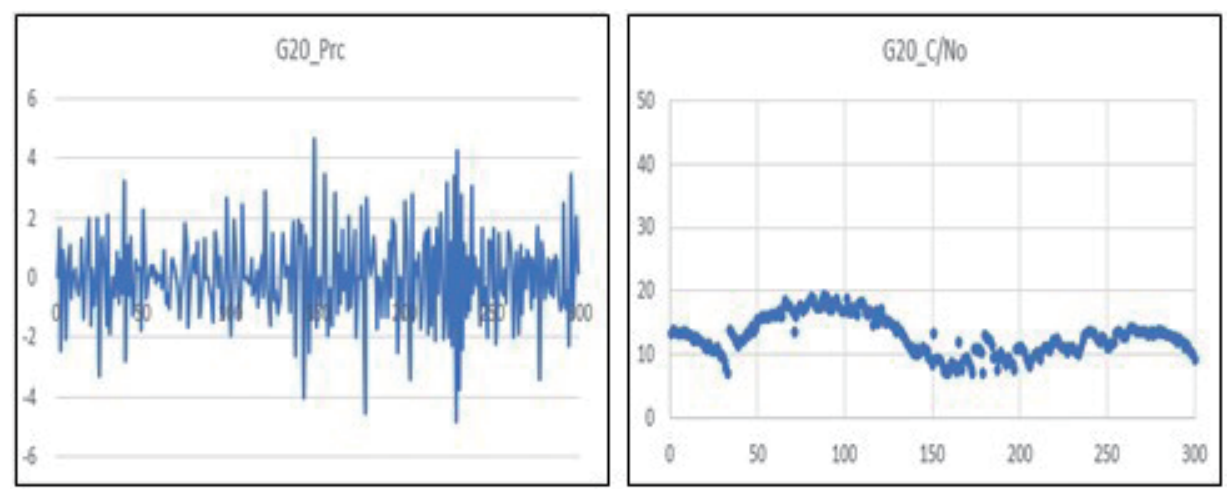

Fig. 10. (Color online) Signal characteristics of the invisible satellites. 


\section{Experiment and Result Analysis}

The experiment was conducted by dividing the research target according to the observation environment into the following types of environment: open terrain with good visibility and environments of low-rise building urban, high-rise building urban, and surrounded by tall buildings. An experiment was conducted to compare and analyze the accuracy by observing three types of smartphone under general conditions. In the remaining three locations, a 3D surface model was built using a UAV, and the visibility range of the smartphone was analyzed using the GIS technique. The visible satellites are classified by analyzing the signal characteristics of the satellites observed by the smartphones, and the final visible satellites are selected by combining them with the visible satellites classified by the sky mask method. The accuracy was verified by comparing the coordinates calculated using the selected visible satellites with the true position coordinates.

\subsection{Experiment and analysis of specified observation environments}

\subsubsection{Open terrain with good visibility}

To validate the GNSS positioning accuracy in an open land without obstacles, each of the four corners of the basketball court was observed for 5 min using three types of smartphone: Samsung Galaxy S8, Xiaomi Mi8, and Xiaomi Mi9. Figure 11 shows the test site, smartphone observation photos, and the ground control point survey method. The coordinates of each corner were acquired by the VRS positioning method using Trimble R8 to verify the accuracy. The VRS value assumed as the ground reference point was compared and analyzed with the location value calculated using the smartphone. Table 3, noted below, shows the observational statistical analysis results. Since it is an open field without any shielding elements, the error calculated
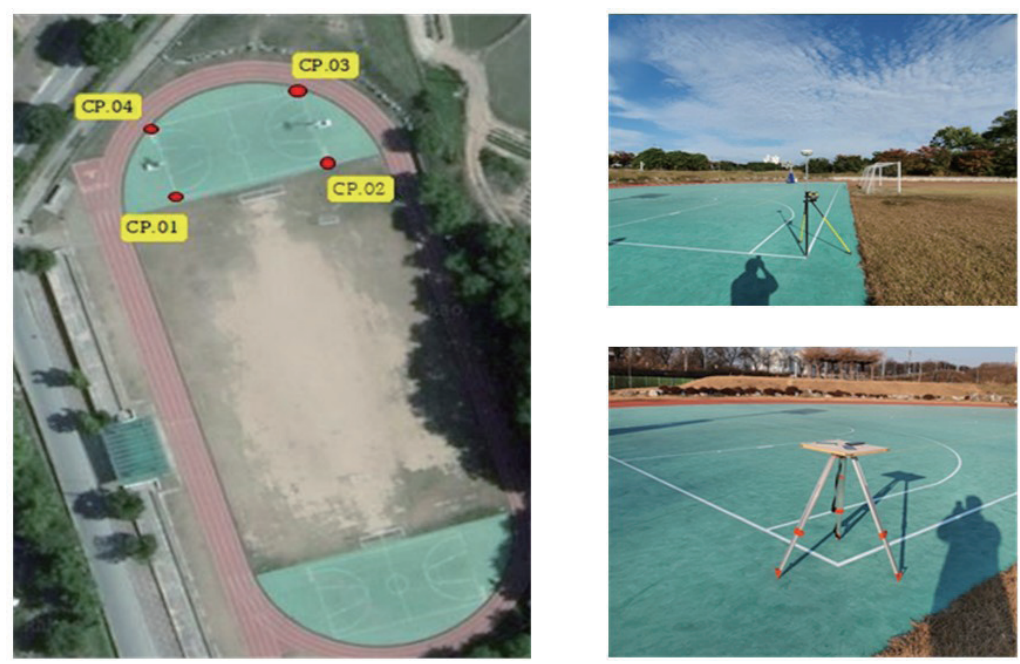

Fig. 11. (Color online) Experimental site: an open field with good visibility. 
Table 3

Open field location analysis, demonstrating statistics with good visibility.

\begin{tabular}{lcccccccc}
\hline & \multicolumn{2}{c}{$\begin{array}{c}\text { CP.01 } \\
\text { (RMSE) }\end{array}$} & \multicolumn{2}{c}{$\begin{array}{c}\text { CP.02 } \\
\text { (RMSE) }\end{array}$} & \multicolumn{2}{c}{$\begin{array}{c}\text { CP.03 } \\
\text { (RMSE) }\end{array}$} & \multicolumn{2}{c}{$\begin{array}{c}\text { CP.04 } \\
\text { (RMSE) }\end{array}$} \\
\cline { 2 - 10 } & $\begin{array}{c}\text { Horiz. } \\
\text { err. (m) }\end{array}$ & $\begin{array}{c}\text { Vert. } \\
\text { err. (m) }\end{array}$ & $\begin{array}{c}\text { Horiz. } \\
\text { err. (m) }\end{array}$ & $\begin{array}{c}\text { Vert. } \\
\text { err. (m) }\end{array}$ & $\begin{array}{c}\text { Horiz. } \\
\text { err. (m) }\end{array}$ & $\begin{array}{c}\text { Vert. } \\
\text { err. (m) }\end{array}$ & $\begin{array}{c}\text { Horiz. } \\
\text { err. (m) }\end{array}$ & $\begin{array}{c}\text { Vert. } \\
\text { err. (m) }\end{array}$ \\
\hline S8 & 0.75 & 2.67 & 1.24 & 2.86 & 0.89 & 1.87 & 2.24 & 2.66 \\
\hline Mi8 & 0.67 & 1.85 & 0.61 & 1.54 & 0.88 & 1.68 & 1.85 & 2.51 \\
\hline Mi9 & 0.71 & 2.35 & 0.74 & 2.28 & 0.74 & 1.45 & 1.81 & 1.89 \\
\hline
\end{tabular}

using each smartphone was derived at the meter level. It was determined that there was no difference between devices; however, in this study, the Xiaomi Mi8 smartphone, which can receive dual-frequency signals and has an advantage in multipath, was used for the rest of the analysis in the study area.

Before the actual experiment, an experiment was conducted to compare the satellite reception rates of a smartphone and a GNSS machine for general surveying according to the observation environment. In general, when an experiment is conducted in the same place, the same type of satellite and satellite signal should be received. However, a difference in satellite signal reception rate is expected since the smartphone uses a low-cost antenna. To determine the satellite signals' reception rate of the two devices, a static survey was conducted using the GNSS survey device Trimble R8 in the same place for relative comparison with the Xiaomi Mi8 smartphone used in this study; the difference in satellite signal reception rate between the two devices was examined (refer to Table 4). The test site was conducted near the Yeungnam University 5 (YU5) station in an environment surrounded by tall buildings where a multipath is expected.

As a result of comparing the two types of satellite, GPS and GLONASS, Trimble R8 received 5 GPS signals and 3 GLONASS signals; however, the smartphone received 9 GPS signals and 4 GLONASS signals. This phenomenon shows that the smartphone's antenna receives both the reflected or diffracted and multipath signals because there is no device to mitigate the multipath. A smartphone antenna is generally a microstrip antenna manufactured using a printed circuit board (PCB); thus, the received signal strength may be lost due to the influence of linear polarization and the directivity of the radiation pattern. An experiment was conducted with reference to the properties of these smartphone antennas.

\subsubsection{Environment of low-rise building urban}

The test area was selected where buildings, trees, and structures were reflected as visibile shielding elements. The experimental areas were designated as Yeungnam University 1, 2, and 3

Table 4

Test results for the signal reception rates.

\begin{tabular}{lcc}
\hline Equipment & Satellite type & Observed satellite signal \\
\hline \multirow{2}{*}{ Trimble R8 } & GPS & PRN10, PRN12, PRN25, PRN31, PRN32 \\
\cline { 2 - 3 } & GLONASS & PRN2, PRN3, PRN12 \\
\hline \multirow{2}{*}{ Mi8 smartphone } & GPS & PRN10, PRN12, PRN20, PRN22, PRN23, PRN25, PRN29, PRN31, PRN32 \\
\cline { 2 - 3 } & GLONASS & PRN2, PRN3, PRN12, PRN13 \\
\hline
\end{tabular}


(YU1, YU2, and YU3) by describing the three campuses of Yeungnam University, respectively. Station YU1 is a place surrounded by buildings and trees, Station YU2 is surrounded by buildings on three sides based on the smartphone's location, and YU3 is surrounded by buildings and trees based on the smartphone's location and the shielding element. This is a place (YU3) where trees can have a significant impact. As shown in Table 5, the visibility range was calculated by GIS analysis, and a sky mask method was performed by the visibility analysis at the smartphone's location. The center point of the sky mask method coincides with the coordinates calculated with the initial position of the smartphone.

Those areas in blue within the sky mask method range are classified as visible satellites, and those areas in red outside the range are classified as invisible satellites (refer to Table 6). Table 7(a) shows the receiving satellites observed with a smartphone. Among the visible satellites according to the sky mask method in Table 6, the satellites for which no satellite signals were observed are not receiving satellites, so Table 7(a) was used to exclude them from the analysis. The results are shown in Table 7(b).

Invisible satellites were classified by calculating the Prc of the satellite signals received from the observed satellites, and the standard deviation of the signal strength and the low altitude of

Table 5

(Color online) Skyline and barrier analyses of YU1, YU2, and YU3 study areas in environment of low-rise building urban.

\begin{tabular}{l}
\hline YU1 \\
\hline Skyline analysis
\end{tabular}


Table 6

(Color online) Satellite ephemeris is used as a sky mask method for the visible range in an environment of low-rise building urban, showing all satellites in the observation timeframe.

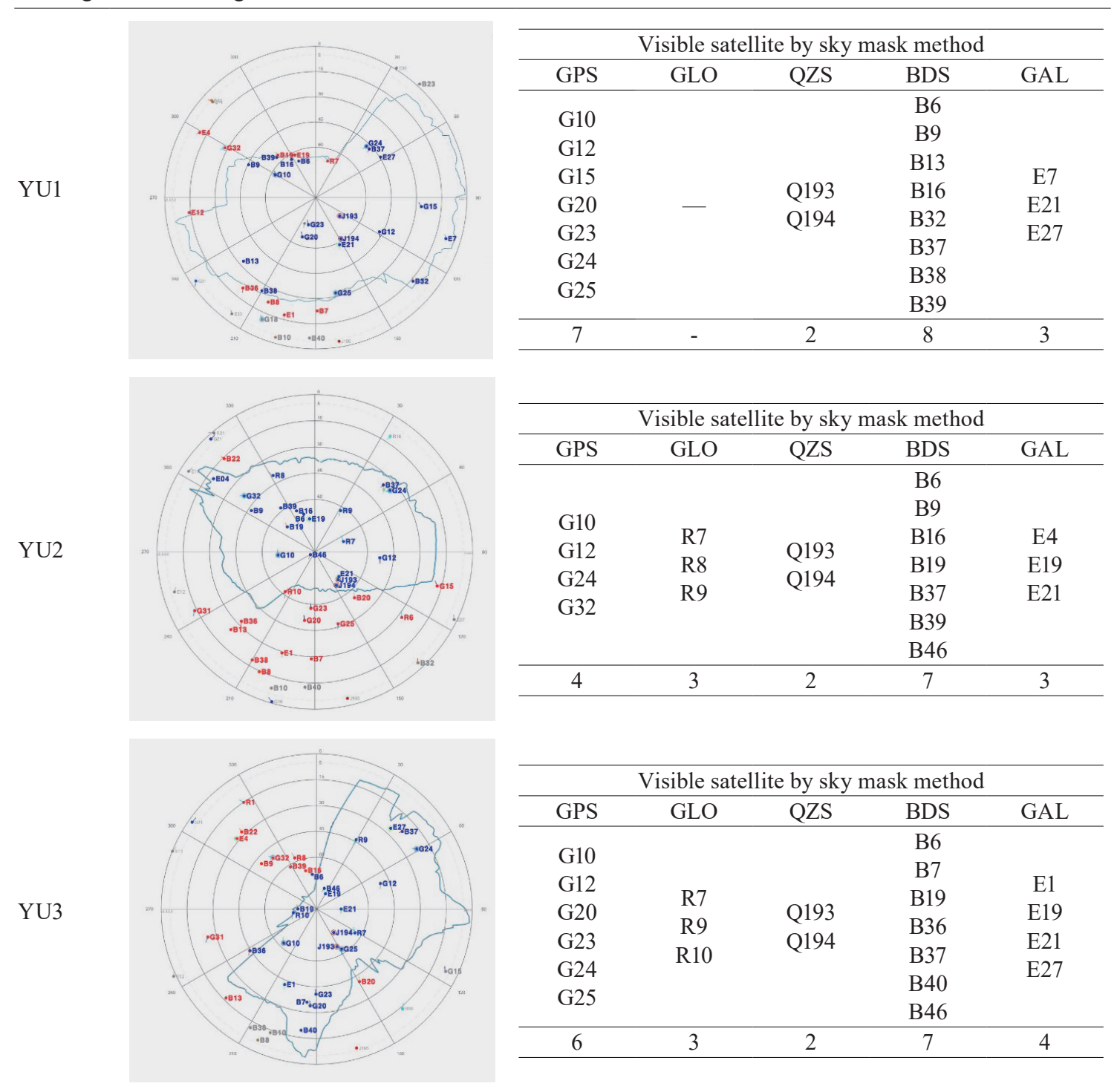

$15^{\circ}$ were excluded using the EA. The average standard deviation of Prc and the receiving satellite signal strength at the YU1 point are 0.256 and 1.944, respectively. The B32 satellite was classified as an invisible satellite owing to its low EA (Table 8).

The average standard deviation of Prc and the receiving satellite signal strength at the YU2 point are 0.264 and 2.399, respectively. The G15 satellites above the average standard deviation were determined to have a Prc standard deviation of 0.913 and a C/No standard deviation of 3.909; thus, they were classified as invisible satellites. Moreover, the G31 and B22 satellites were classified as invisible satellites owing to their low EAs.

The average standard deviation of Prc and the receiving satellite signal strength at the YU3 point are 0.148 and 1.937, respectively. The B16 satellites above the average standard deviation 
Table 7

Satellite types for study areas YU1, YU2, and YU3 in environment of low-rise building urban: (a) receiving satellites and (b) visible satellites by sky mask method.

\begin{tabular}{|c|c|c|c|c|c|c|c|c|c|c|}
\hline & & & (a) & & & & & (b) & & \\
\hline & GPS & GLO & QZS & BDS & GAL & GPS & GLO & QZS & BDS & GAL \\
\hline \multirow[t]{2}{*}{ YU1 } & $\begin{array}{l}\text { G10 } \\
\text { G12 } \\
\text { G15 } \\
\text { G20 } \\
\text { G23 } \\
\text { G24 } \\
\text { G25 }\end{array}$ & $\mathrm{R} 7$ & $\begin{array}{l}\text { Q193 } \\
\text { Q194 }\end{array}$ & $\begin{array}{l}\text { B6 } \\
\text { B8 } \\
\text { B9 } \\
\text { B16 } \\
\text { B19 } \\
\text { B32 } \\
\text { B36 } \\
\text { B37 }\end{array}$ & $\begin{array}{l}\text { E19 } \\
\text { E21 }\end{array}$ & $\begin{array}{l}\text { G10 } \\
\text { G12 } \\
\text { G15 } \\
\text { G20 } \\
\text { G23 } \\
\text { G24 } \\
\text { G25 }\end{array}$ & - & $\begin{array}{l}\text { Q193 } \\
\text { Q194 }\end{array}$ & $\begin{array}{c}\text { B6 } \\
\text { B9 } \\
\text { B16 } \\
\text { B32 } \\
\text { B37 }\end{array}$ & E21 \\
\hline & 7 & 1 & 2 & 8 & 2 & 7 & - & 2 & 5 & 1 \\
\hline \multirow{3}{*}{ YU2 } & GPS & GLO & QZS & BDS & GAL & GPS & GLO & QZS & BDS & GAL \\
\hline & $\begin{array}{l}\text { G10 } \\
\text { G12 } \\
\text { G15 } \\
\text { G20 } \\
\text { G23 } \\
\text { G24 } \\
\text { G31 } \\
\text { G32 }\end{array}$ & $\begin{array}{l}\text { R6 } \\
\text { R7 } \\
\text { R9 }\end{array}$ & $\begin{array}{l}\text { Q193 } \\
\text { Q194 }\end{array}$ & $\begin{array}{c}\text { B6 } \\
\text { B8 } \\
\text { B9 } \\
\text { B16 } \\
\text { B19 } \\
\text { B22 } \\
\text { B36 } \\
\text { B37 }\end{array}$ & $\begin{array}{l}\text { E19 } \\
\text { E21 }\end{array}$ & $\begin{array}{l}\text { G10 } \\
\text { G12 } \\
\text { G24 } \\
\text { G32 }\end{array}$ & $\begin{array}{l}\text { R7 } \\
\text { R9 }\end{array}$ & $\begin{array}{l}\text { Q193 } \\
\text { Q194 }\end{array}$ & $\begin{array}{c}\text { B6 } \\
\text { B9 } \\
\text { B16 } \\
\text { B19 } \\
\text { B37 }\end{array}$ & $\begin{array}{l}\text { E19 } \\
\text { E21 }\end{array}$ \\
\hline & 8 & 3 & 2 & 8 & 2 & 4 & 2 & 2 & 5 & 2 \\
\hline \multirow{3}{*}{ YU3 } & GPS & GLO & QZS & BDS & GAL & GPS & GLO & QZS & BDS & GAL \\
\hline & $\begin{array}{l}\text { G10 } \\
\text { G15 } \\
\text { G20 } \\
\text { G23 } \\
\text { G24 } \\
\text { G25 } \\
\text { G31 } \\
\text { G32 }\end{array}$ & $\begin{array}{l}\text { R1 } \\
\text { R7 } \\
\text { R9 }\end{array}$ & $\begin{array}{l}\text { Q193 } \\
\text { Q194 }\end{array}$ & $\begin{array}{c}\text { B6 } \\
\text { B9 } \\
\text { B10 } \\
\text { B16 } \\
\text { B19 } \\
\text { B22 } \\
\text { B36 } \\
\text { B37 }\end{array}$ & - & $\begin{array}{l}\text { G10 } \\
\text { G20 } \\
\text { G23 } \\
\text { G24 } \\
\text { G25 }\end{array}$ & $\begin{array}{l}\text { R7 } \\
\text { R9 }\end{array}$ & $\begin{array}{l}\text { Q193 } \\
\text { Q194 }\end{array}$ & $\begin{array}{c}\text { B6 } \\
\text { B19 } \\
\text { B36 } \\
\text { B37 }\end{array}$ & - \\
\hline & 8 & 3 & 2 & 8 & - & 5 & 2 & 2 & 4 & - \\
\hline
\end{tabular}

were determined to have a Prc standard deviation of 0.786 and a C/No standard deviation of 3.426; thus, they were classified as invisible satellites. Moreover, the G15, R1, and B10 satellites were classified as invisible satellites owing to their low EAs.

\subsubsection{Environment of high-rise building urban}

The high-rise building urban experimental area was in Andong-si, Gyeongsangbuk-do, where the new city was formed, and the experiment was conducted by selecting a point with a narrow road between buildings. Visibile shielding elements are mainly buildings and other structures, and the multipath is expected to be severe because the exterior walls of the buildings are made of reflective materials such as glass. The experimental target areas were designated as Andong-si 1, 2, and 3 (A1, A2, and A3, respectively) by designating three downtown areas near the Andong-si New Provincial Office. A1 is a place where the buildings are formed on both 
Table 8

Visible satellites classified by analysis of the signal characteristics in environment of low-rise building urban: (a) Prc and (b) C/No.

\begin{tabular}{|c|c|c|c|c|c|c|}
\hline \multirow{2}{*}{ Satellite No. } & \multicolumn{3}{|c|}{ (a) } & \multicolumn{3}{|c|}{ (b) } \\
\hline & YU1 & YU2 & YU3 & YU1 & YU2 & YU3 \\
\hline G10 & 0.221 & 0.187 & 0.104 & 1.193 & 4.952 & 0.889 \\
\hline G12 & 0.222 & 0.159 & - & 0.964 & 0.644 & 一 \\
\hline G15 & 0.229 & 0.913 & ** & 5.504 & 3.909 & ** \\
\hline G20 & 0.222 & 0.229 & 0.110 & 1.052 & 1.983 & 3.217 \\
\hline G23 & 0.222 & 0.289 & 0.103 & 1.221 & 4.534 & 2.406 \\
\hline G24 & 0.225 & 0.159 & 0.114 & 1.488 & 2.149 & 2.089 \\
\hline G25 & $0.381^{*}$ & - & 0.104 & $4.878\left(^{*}\right)$ & - & 1.041 \\
\hline G31 & - & ** & 0.136 & - & ** & 2.846 \\
\hline G32 & - & - & 0.103 & 一 & - & 3.395 \\
\hline $\mathrm{R} 1$ & - & - & $* *$ & - & - & $* *$ \\
\hline R6 & - & $0.571^{*}$ & - & - & $3.951^{*}$ & - \\
\hline R7 & 0.221 & 0.159 & 0.103 & 0.826 & 0.666 & 0.983 \\
\hline R9 & & 0.159 & 0.104 & - & 1.404 & 1.163 \\
\hline Q193 & 0.224 & 0.159 & 0.103 & 1.214 & 0.151 & 0.815 \\
\hline Q194 & 0.224 & 0.159 & 0.103 & 0.382 & 0.349 & 0.596 \\
\hline B6 & 0.224 & 0.160 & 0.129 & 0.727 & 0.787 & 1.854 \\
\hline B8 & $0.582^{*}$ & $0.679^{*}$ & - & $5.423^{*}$ & $6.757^{*}$ & - \\
\hline B9 & 0.230 & 0.160 & 0.103 & 1.795 & 1.685 & 0.671 \\
\hline $\mathrm{B} 10$ & - & - & ** & - & - & ** \\
\hline B16 & 0.225 & 0.159 & $0.786^{*}$ & 0.888 & 0.341 & $3.426^{*}$ \\
\hline B19 & 0.224 & 0.159 & 0.104 & 1.148 & 0.957 & 0.596 \\
\hline B22 & - & $* *$ & 0.121 & - & $* *$ & 2.301 \\
\hline B32 & ** & - & - & ** & - & - \\
\hline B36 & $0.296^{*}$ & $0.401^{*}$ & 0.104 & $4.066^{*}$ & $5.452^{*}$ & 3.469 \\
\hline B37 & 0.224 & 0.205 & 0.122 & 0.879 & 5.612 & 3.114 \\
\hline E19 & 0.225 & 0.160 & - & 0.588 & 0.763 & - \\
\hline E21 & 0.250 & 0.160 & - & 2.693 & 0.528 & - \\
\hline Average & 0.256 & 0.264 & 0.148 & 1.944 & 2.399 & 1.937 \\
\hline
\end{tabular}

*Invisible satellite

${ }^{* *}$ Satellites with an EA of $15^{\circ}$ or less

sides, and there is a narrow alley. A2 is a location where the visibility is secured from northeast to southwest as opposed to the A1 station; A2 is located between buildings, so the visibility range is not wide and multipath effects are expected. A3 is a location where visibility is secured from northwest to southeast, and the apartment is located to the north between the buildings, so the visibility range is not wide and multipath effects are expected. In the environment of highrise building urban, as in the environment of low-rise building urban, the visibility range was calculated by GIS analysis for the visibility analysis at the smartphone location, and the sky mask method was performed (refer to Table 9).

A1 is located between buildings, so it has high shielding, and visibility is secured only to the south and north. Thus, A1 is a place where only a specific range of satellites can be received. A2 is located on one side between buildings, has high shielding, and visibility only to the east and west, so only a certain range of satellites can be received. A3 is located between buildings, and 
Table 9

(Color online) Skyline and barrier analysis methods of A1, A2, and A3 study areas in environment of high-rise building urban.

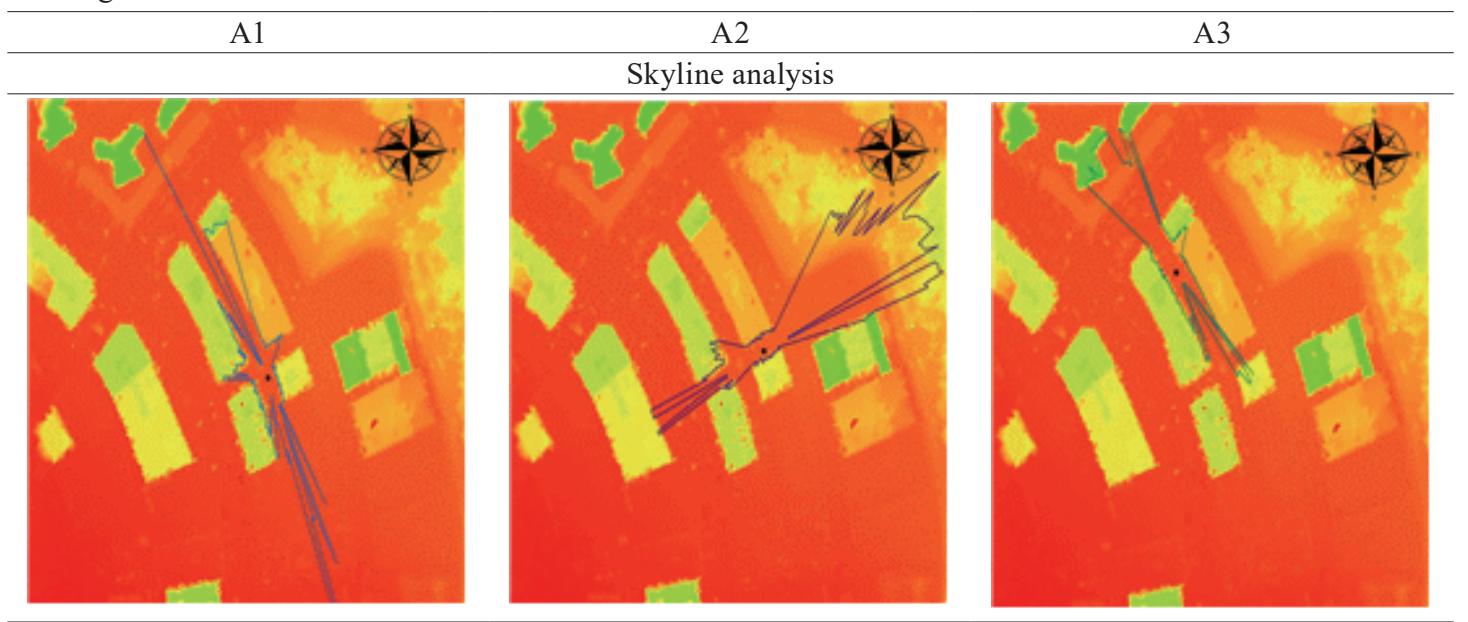

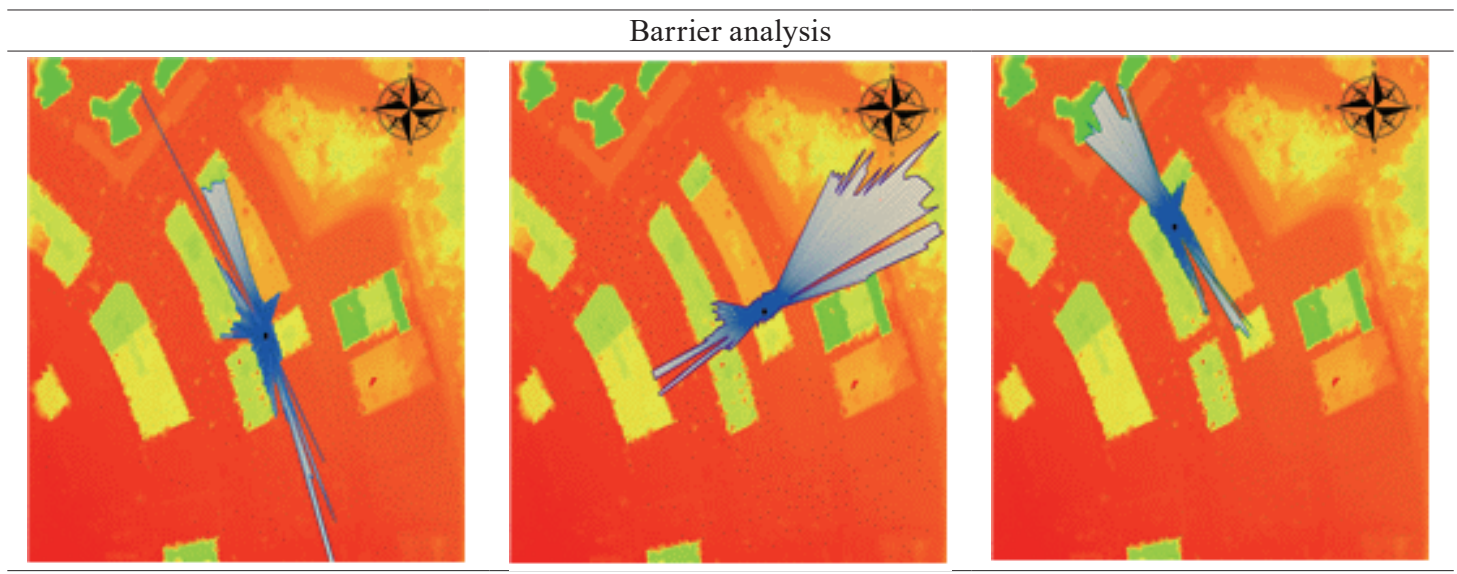

the visible range was calculated differently because the heights of both buildings are different. All three locations are places where the surrounding buildings can only receive a certain range of satellites. Except for the high-altitude satellites, signals from other satellites are determined to be multipath. As in the environment of low-rise building urban, the satellites in blue within the celestial range are classified as visible satellites, and the satellites in red outside the range are classified as invisible satellites. Table 10(a) shows the receiving satellites observed with a smartphone. The visible satellites according to the sky mask method are shown in Table 11, and the satellites for which no satellite signals were observed are not receiving satellites, so Table 10(a) was used to exclude them from the analysis. The results are shown in Table 10(b).

The signal characteristics of the observed satellites were analyzed in the same way as in an environment of low-rise building urban. The average standard deviation of Prc and the receiving satellite signal strength of A1 are 0.227 and 3.213, respectively (Table 12).

The G24 satellites with an average standard deviation or higher were determined to have a Prc standard deviation of 0.553 and a C/No standard deviation of 6.619; thus, they were classified as invisible satellites. The average standard deviation of Prc and the receiving satellite signal 
Table 10

(Color online) Satellite ephemeris is used as a sky mask method for the visible range in an environment of high-rise building urban, showing all satellites in the observation timeframe.

A1

A2

A3
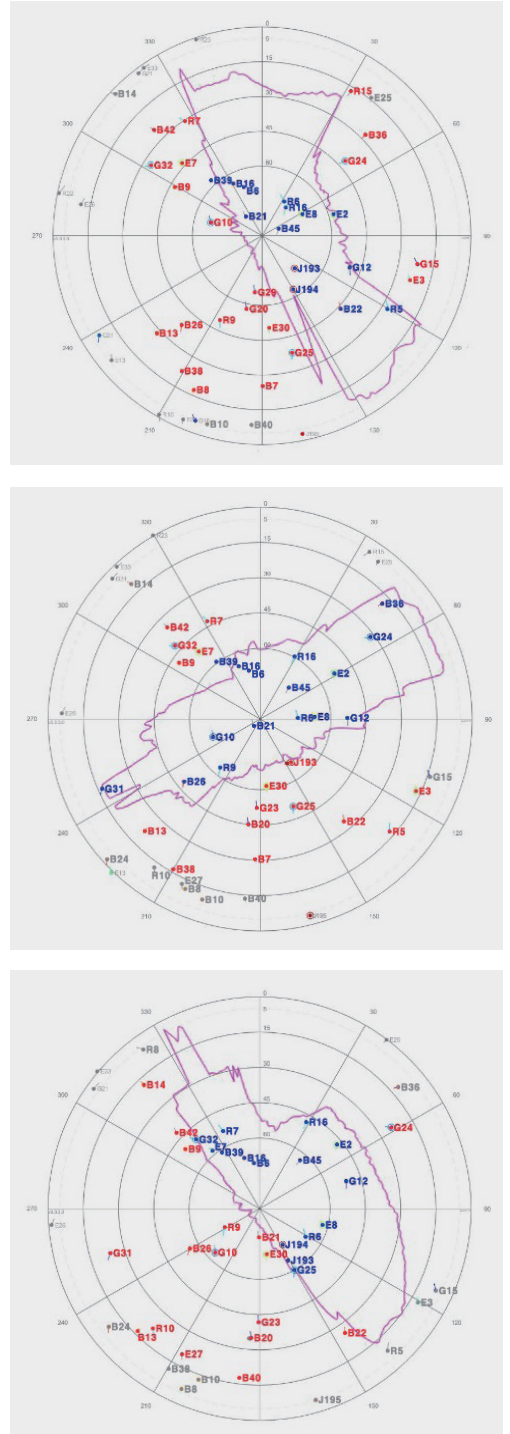

\begin{tabular}{ccccc}
\hline \multicolumn{5}{c}{ Visible satellite by sky mask method } \\
\hline GPS & GLO & QZS & BDS & GAL \\
\hline \multirow{4}{*}{ G12 } & & & B6 & \\
& R6 & & B16 & \\
& R5 & Q193 & B21 & E2 \\
& R16 & Q194 & B22 & E8 \\
& & & B39 & \\
\hline 1 & 3 & 2 & 6 & 2 \\
\hline
\end{tabular}

\begin{tabular}{ccccc}
\hline \multicolumn{5}{c}{ Visible satellite by sky mask method } \\
\hline GPS & GLO & QZS & BDS & GAL \\
\hline & & & B6 & \\
G10 & R6 & & B16 & \\
G12 & B21 & - & B26 & E2 \\
G24 & R16 & & B36 & E8 \\
G31 & & & B39 & \\
& & & B45 & \\
\hline 4 & 3 & - & 7 & 2 \\
\hline
\end{tabular}

\begin{tabular}{ccccc}
\hline \multicolumn{5}{c}{ Visible satellite by sky mask method } \\
\hline GPS & GLO & QZS & BDS & GAL \\
\hline \multirow{2}{*}{ G12 } & R6 & & B6 & \multirow{2}{*}{ Q2 } \\
G25 & R7 & Q193 & B16 & B79 \\
G32 & R16 & & B45 & E8 \\
\hline 3 & 3 & 2 & 4 & 3 \\
\hline
\end{tabular}

strength of A2 are 0.224 and 3.392, respectively. Furthermore, the G12 satellites above the average standard deviation were determined to have a Prc standard deviation of 0.353 and a $\mathrm{C} /$ No standard deviation of 5.135; thus, they were classified as invisible satellites. In addition, the G31, B8, B10, and E3 satellites were classified as invisible satellites owing to their low EAs. The average standard deviation of Prc and the receiving satellite signal strength of A3 are 0.317 and 3.037, respectively. The G23 satellites above the average standard deviation were determined to have a Prc standard deviation of 0.758 and a C/No standard deviation of 3.910; thus, they were classified as invisible satellites. Moreover, the G15 and B36 satellites were classified as invisible satellites owing to their low EAs. 
Table 11

Satellite types according to sky mask method for study areas A1, A2, and A3 in environment of high-rise building urban: (a) receiving satellites and (b) visible satellites.

\begin{tabular}{|c|c|c|c|c|c|c|c|c|c|c|}
\hline & & & (a) & & & & & (b) & & \\
\hline \multirow{10}{*}{ YU1 } & GPS & GLO & QZS & BDS & GAL & GPS & GLO & QZS & BDS & GAL \\
\hline & G10 & \multirow{8}{*}{ R6 } & \multirow{8}{*}{ - } & \multirow{8}{*}{$\begin{array}{c}\text { B9 } \\
\text { B16 } \\
\text { B26 } \\
\text { B36 }\end{array}$} & \multirow{8}{*}{$\begin{array}{l}\text { E2 } \\
\text { E7 } \\
\text { E8 }\end{array}$} & \multirow{8}{*}{ G12 } & \multirow{8}{*}{ R6 } & \multirow{8}{*}{-} & \multirow{8}{*}{ B16 } & \multirow{8}{*}{$\begin{array}{l}\text { E2 } \\
\text { E8 }\end{array}$} \\
\hline & G12 & & & & & & & & & \\
\hline & G15 & & & & & & & & & \\
\hline & G20 & & & & & & & & & \\
\hline & G23 & & & & & & & & & \\
\hline & G24 & & & & & & & & & \\
\hline & G25 & & & & & & & & & \\
\hline & G32 & & & & & & & & & \\
\hline & 8 & 1 & - & 4 & 3 & 1 & 1 & - & 1 & 2 \\
\hline \multirow{11}{*}{ YU2 } & GPS & GLO & QZS & BDS & GAL & GPS & GLO & QZS & BDS & GAL \\
\hline & G10 & & \multirow{9}{*}{ Q193 } & \multirow{9}{*}{$\begin{array}{c}\text { B8 } \\
\text { B9 } \\
\text { B10 } \\
\text { B13 } \\
\text { B16 } \\
\text { B22 } \\
\text { B26 } \\
\text { B36 }\end{array}$} & \multirow{9}{*}{$\begin{array}{c}\text { E2 } \\
\text { E3 } \\
\text { E7 } \\
\text { E8 } \\
\text { E30 }\end{array}$} & & \multirow{9}{*}{$\begin{array}{c}\text { R6 } \\
\text { R9 } \\
\text { R16 }\end{array}$} & \multirow{9}{*}{-} & \multirow{9}{*}{$\begin{array}{l}\text { B16 } \\
\text { B26 } \\
\text { B36 }\end{array}$} & \multirow{9}{*}{$\begin{array}{l}\text { E2 } \\
\text { E8 }\end{array}$} \\
\hline & G12 & & & & & & & & & \\
\hline & G15 & R5 & & & & & & & & \\
\hline & G20 & R6 & & & & G12 & & & & \\
\hline & G23 & R7 & & & & Gl2 & & & & \\
\hline & G24 & R9 & & & & G24 & & & & \\
\hline & G25 & R16 & & & & & & & & \\
\hline & G31 & & & & & & & & & \\
\hline & $\mathrm{G} 32$ & & & & & & & & & \\
\hline & 9 & 5 & 1 & 8 & 5 & 4 & 3 & - & 3 & 2 \\
\hline \multirow{11}{*}{ YU3 } & GPS & GLO & QZS & BDS & GAL & GPS & GLO & QZS & BDS & GAL \\
\hline & G10 & & \multirow{9}{*}{$\begin{array}{l}\text { Q193 } \\
\text { Q194 }\end{array}$} & \multirow{9}{*}{$\begin{array}{c}\text { B9 } \\
\text { B16 } \\
\text { B22 } \\
\text { B26 } \\
\text { B36 }\end{array}$} & \multirow{9}{*}{$\begin{array}{c}\text { E2 } \\
\text { E8 } \\
\text { E30 }\end{array}$} & & \multirow{9}{*}{$\begin{array}{c}\text { R6 } \\
\text { R7 } \\
\text { R16 }\end{array}$} & \multirow{9}{*}{$\begin{array}{l}\text { Q193 } \\
\text { Q194 }\end{array}$} & \multirow{9}{*}{ B16 } & \multirow{9}{*}{$\begin{array}{l}\text { E2 } \\
\text { E8 }\end{array}$} \\
\hline & G12 & & & & & & & & & \\
\hline & G15 & R6 & & & & & & & & \\
\hline & G20 & R7 & & & & G12 & & & & \\
\hline & G23 & R9 & & & & G25 & & & & \\
\hline & $\mathrm{G} 24$ & R10 & & & & G32 & & & & \\
\hline & G25 & R16 & & & & & & & & \\
\hline & G31 & & & & & & & & & \\
\hline & G32 & & & & & & & & & \\
\hline & 9 & 5 & 2 & 5 & 3 & 3 & 3 & 2 & 1 & 2 \\
\hline
\end{tabular}

\subsubsection{Environment surrounded by tall buildings}

YU5 is an environment surrounded by tall buildings with a structure blocked by buildings with a height of $20 \mathrm{~m}$ or more on all sides of the station, and the visibility range is relatively narrow; therefore, it is challenging to obtain visible satellite signals. The sky mask method was developed by calculating the visible range through GIS analysis, and it can be seen that a considerable number of satellite signals outside the analyzed visible range were received. This result is expected to be a multipath error because many factors cause signal reflection around the YU5's spatial structure, and the low-angle satellite signals are expected to be secondary and tertiary multipath signals owing to the exterior wall of the building (Tables 13-15).

The averages of the Prc and strength of the receiving satellite signal at YU5 are 0.420 and 3.489 , respectively. The G10 satellites above the average standard deviation were determined to have a Prc standard deviation of 0.481 and a C/No standard deviation of 3.940; thus, they were classified as invisible satellites (Table 16). 
Table 12

Visible satellites classified by analysis of the following signal characteristics in environment of high-rise building urban: (a) Prc and (b) C/No.

\begin{tabular}{|c|c|c|c|c|c|c|}
\hline \multirow{2}{*}{ Satellite No. } & \multicolumn{3}{|c|}{ (a) } & \multicolumn{3}{|c|}{ (b) } \\
\hline & A1 & A2 & A3 & A1 & A2 & A3 \\
\hline G10 & 0.091 & 0.122 & 0.118 & 1.342 & 3.118 & 2.879 \\
\hline G12 & 0.093 & $0.353^{*}$ & 0.212 & 2.623 & $5.135^{*}$ & 5.675 \\
\hline G15 & 0.092 & $0.401^{*}$ & $* *$ & 3.195 & $7.062^{*}$ & $* *$ \\
\hline G20 & 0.091 & 0.123 & 0.312 & 1.652 & 1.990 & 3.397 \\
\hline G23 & 0.091 & 0.123 & $0.758^{*}$ & 1.584 & 3.575 & $3.910^{*}$ \\
\hline G24 & $0.553^{*}$ & 0.122 & 0.116 & $6.619^{*}$ & 1.352 & 3.255 \\
\hline G25 & 0.091 & 0.124 & 0.116 & 2.091 & 2.655 & 0.963 \\
\hline G31 & - & $* *$ & 0.268 & - & $* *$ & 5.835 \\
\hline G32 & $0.597^{*}$ & 0.206 & 0.115 & $6.729^{*}$ & 4.937 & 1.986 \\
\hline R5 & - & $0.261^{*}$ & - & - & $5.392^{*}$ & - \\
\hline R6 & 0.093 & 0.122 & 0.119 & 1.02 & 0.553 & 3.821 \\
\hline R7 & - & 0.149 & 0.116 & - & 5.152 & 1.488 \\
\hline R9 & - & 0.122 & $0.869^{*}$ & - & 2.444 & $4.136^{*}$ \\
\hline R10 & - & - & $1.235^{*}$ & - & - & $4.969^{*}$ \\
\hline R16 & - & 0.209 & 0.265 & - & 5.020 & 6.057 \\
\hline Q193 & - & 0.149 & 0.116 & - & 1.670 & 0.567 \\
\hline Q194 & - & - & 0.116 & - & - & 0.432 \\
\hline B8 & - & $* *$ & - & - & $* *$ & - \\
\hline B9 & $1.003^{*}$ & 0.122 & 0.116 & $4.353^{*}$ & 1.044 & 1.109 \\
\hline B10 & - & $* *$ & - & - & $* *$ & - \\
\hline B13 & - & 0.382 & - & - & 2.212 & - \\
\hline B16 & 0.091 & 0.121 & 0.116 & 0.705 & 1.472 & 0.416 \\
\hline B22 & - & $0.760^{*}$ & 0.135 & - & $4.582^{*}$ & 3.867 \\
\hline B26 & 0.150 & 0.121 & 0.279 & 4.751 & 1.597 & 3.930 \\
\hline B36 & $0.565^{*}$ & 0.122 & $* *$ & $5.907^{*}$ & 3.568 & $* *$ \\
\hline E2 & 0.092 & 0.122 & 0.116 & 2.575 & 1.207 & 1.753 \\
\hline E3 & - & $* *$ & - & - & ** & - \\
\hline E7 & $0.650^{*}$ & $0.649^{*}$ & - & $3.831^{*}$ & $6.149^{*}$ & - \\
\hline E8 & 0.091 & 0.122 & $1.235^{*}$ & 2.426 & 2.486 & $5.177^{*}$ \\
\hline E30 & - & $0.270^{*}$ & 0.116 & - & $7.024^{*}$ & 1.184 \\
\hline Average & 0.277 & 0.224 & 0.317 & 3.213 & 3.392 & 3.037 \\
\hline
\end{tabular}

*Invisible satellite

${ }^{* *}$ Satellites with an elevation angle of $15^{\circ}$ or less

Table 13

(Color online) Skyline and barrier analysis of YU5 study area in an environment surrounded by tall buildings.

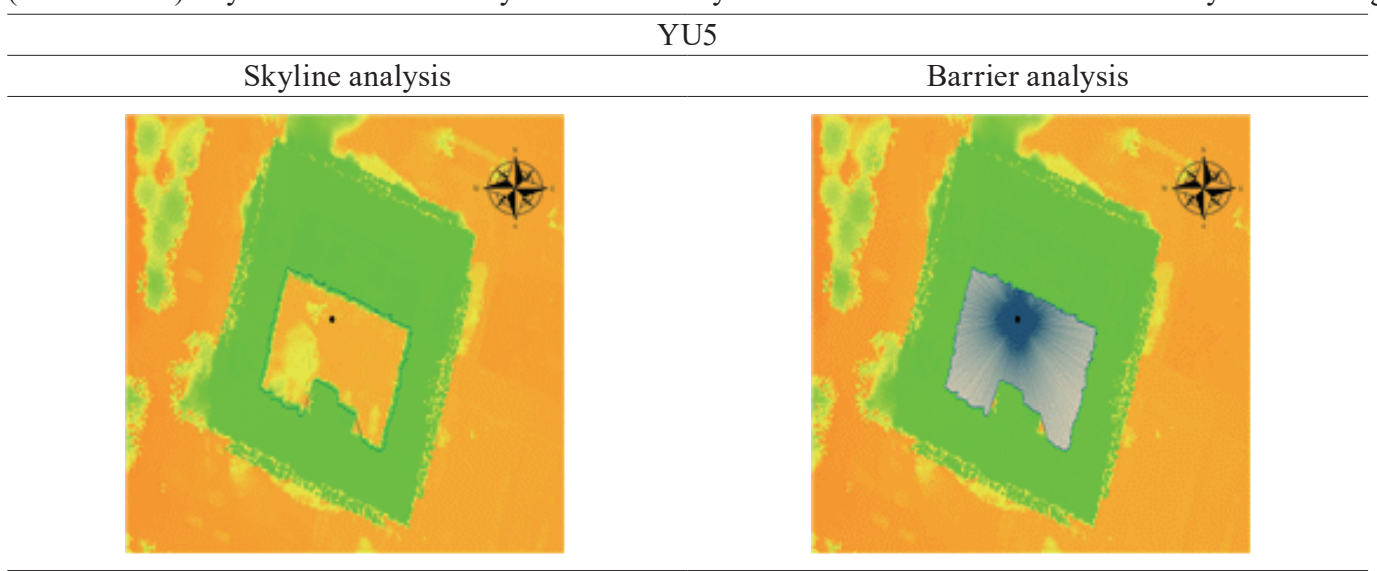


Table 14

(Color online) Satellite ephemeris is used as a sky mask method for the visible range in an environment surrounded by tall buildings, showing all satellites in the observation timeframe.

\begin{tabular}{l}
\multicolumn{5}{c}{ Visible satellite by sky mask method } \\
\cline { 2 - 6 } \\
\cline { 3 - 6 }
\end{tabular}

Table 15

Satellite types according to sky mask method for study area YU5 in an environment surrounded by tall buildings: (a) receiving satellites and (b) visible satellites.

\begin{tabular}{|c|c|c|c|c|c|c|c|c|c|c|}
\hline & \multicolumn{5}{|c|}{ (a) } & \multicolumn{5}{|c|}{ (b) } \\
\hline & GPS & GLO & QZS & BDS & GAL & GPS & GLO & QZS & BDS & GAL \\
\hline \multirow{10}{*}{ YU5 } & G10 & & & B6 & & & & & & \\
\hline & G12 & & & B9 & & & & & & \\
\hline & G20 & & & B10 & & & & & B6 & \\
\hline & G22 & $\begin{array}{l}\mathrm{R} 2 \\
\mathrm{R} 3\end{array}$ & & B16 & & G25 & $\mathrm{R} 2$ & & B9 & \\
\hline & G23 & R3 & Q193 & B19 & E7 & G31 & $\mathrm{R} 12$ & Q193 & B16 & E7 \\
\hline & G25 & R12 & Q194 & B20 & E27 & G32 & $\mathrm{R} 13$ & Q194 & B19 & E27 \\
\hline & G29 & R13 & & B28 & & & & & B37 & \\
\hline & G31 & & & B36 & & & & & & \\
\hline & G32 & & & B37 & & & & & & \\
\hline & 9 & 4 & 2 & 9 & 2 & 3 & 3 & 2 & 5 & 2 \\
\hline
\end{tabular}

\subsection{Final analysis of smartphone GNSS accuracy via visible satellite combinations}

The research site was selected for the smartphone GNSS positioning accuracy test based on satellite visibility, and the experiment was conducted. An optimal combination of visible satellites, consisting of the satellite predicted as a visible satellite by the sky mask method and the observed visible satellite classified by the analysis of the signal characteristics, was utilized. The satellites commonly classified by the two classification methods are called common satellites. Some satellites were classified as visible satellites in the analysis of the signal characteristics but were not classified as candidate satellites in the satellite combination. To this end, the visible satellites were reclassified through correlation analysis with common satellites using the Prc residuals of the satellite signals. The position calculation via GNSS utilized as many satellites as possible, thereby increasing the positioning accuracy. Therefore, in the determination of the correlation between the common and candidate satellites, when the average correlation coefficient was 0.4 or more, the satellite was classified as a visible satellite (Fig. 12). In this study, we calculated the coordinates by analyzing satellite signals, and satellites that have not received satellite signals were excluded from the calculation. If the satellite was excluded 
Table 16

Visible satellites classified by analysis of the following signal characteristics in an environment surrounded by tall buildings: (a) Prc and (b) C/No.

\begin{tabular}{lcc}
\hline Satellite No. & $(\mathrm{a})$ & $(\mathrm{b})$ \\
\hline G10 & $0.481^{*}$ & $3.940^{*}$ \\
G12 & 0.323 & 5.073 \\
G20 & $* *$ & $* *$ \\
G22 & $* *$ & $* *$ \\
G23 & $* *$ & 2.748 \\
G25 & 0.220 & $3.590^{*}$ \\
G29 & $0.695^{*}$ & 1.369 \\
G31 & 0.174 & 2.576 \\
G32 & 0.174 & 0.986 \\
R2 & 0.173 & 3.186 \\
R3 & 0.637 & $3.603^{*}$ \\
R12 & $0.502^{*}$ & $5.452^{*}$ \\
R13 & $0.763^{*}$ & 2.407 \\
Q193 & 0.217 & 0.844 \\
Q194 & 0.173 & $5.585^{*}$ \\
B6 & $0.774^{*}$ & $4.857^{*}$ \\
B9 & $0.604^{*}$ & $5.468^{*}$ \\
B10 & $4.275^{*}$ \\
B16 & $5.726^{*}$ & $3.920^{*}$ \\
B19 & $0.938^{*}$ & $* .434$ \\
B20 & $0.531^{*}$ & $* .489$ \\
B28 & 0.188 & 2.691 \\
B36 & $* * * 263$ \\
B37 & $* *$ & 1.010 \\
E7 & & 3.489 \\
E27 & 0.188 & \\
\hline Average & 0.174 & \\
\hline & 0.174 & \\
\hline
\end{tabular}

\section{Correlation coefficient}

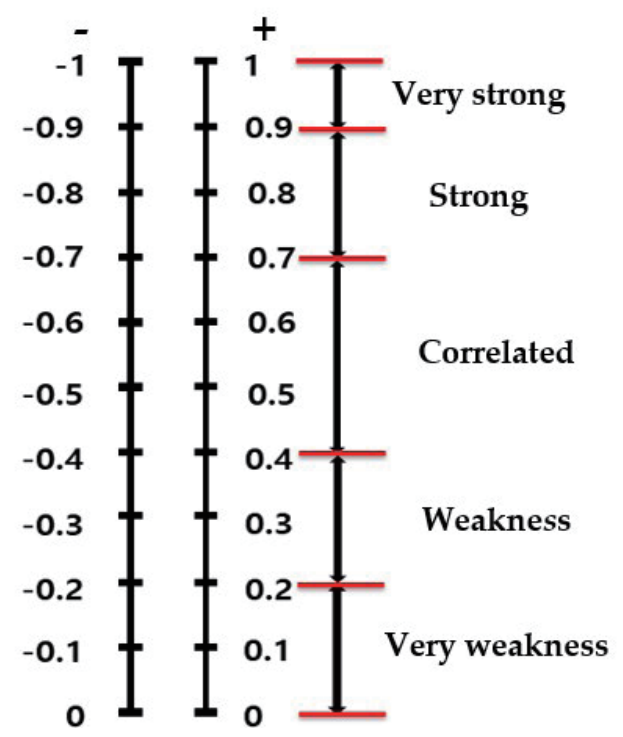

Fig. 12. (Color online) Correlation coefficient analysis of common and candidate satellites. 
from the analysis of the signal characteristics, the satellite would also be excluded from the final satellite classification, even if it was a visible satellite as predicted by the sky mask method. This feature can be a method of reclassifying the misclassified visible satellites into invisible satellites, resulting from the inaccurate sky mask method analysis due to the initial position error of the smartphone.

\subsubsection{Environment of low-rise building urban}

The YU1 study site had 13 commonly visible satellites analyzed by the signal characteristic classification method. Among the visible satellites according to the sky chart, G25 and B32 are not common satellites and are excluded. In the signal characteristic classification, R7, B19, and E19 are not common satellites; however, they were selected as the final visible satellites because their average correlation coefficients were $0.98,0.99$, and 0.98 in the correlation analysis, consistent with the case of the commonly visible satellites. For the YU2 study site, 15 commonly visible satellites were analyzed by the signal characteristic classification method. G20 was not a common satellite in the signal characteristic classification, yet was selected as a final visible satellite because its average correlation coefficient was 0.66 in the correlation analysis, consistent with the case of commonly visible satellites. For the YU3 study site, 13 commonly visible satellites were analyzed by the signal characteristic classification method. G31, G32, B9, and B22 were not common satellites as determined by the signal characteristic classification method; however, they were selected as the final visible satellites because their average correlation coefficients were $0.72,0.95,0.95$, and 0.79 in the correlation analysis, consistent with the case of the commonly visible satellites (refer to Table 17).

Accuracy was calculated on the basis of VRS values. The WLS method provides the standard deviation ( $\sigma$ ) of Prc or $C / N o$ for the raw data of the smartphone. The WLS method (not the least squares method) was performed using $\sigma$ by setting the pseudorange standard deviation as weight. The WLS values in the table are calculated by the WLS method using all satellite signals received from the smartphone, and the SM technique is used in this study. A coordinate calculation value is calculated by the WLS method by considering Eq. (7), which shows the rate of improvement in Table 18.

$$
\text { Rate of improvement }(\%)=\frac{W L S-S M \text { method }}{S M \text { method }} \times 100
$$

As a result of the SM technique experiment in an environment of low-rise building urban, the overall rate of improvement was about 285 to $880 \%$. The distance errors of the WLS for the true value were 2.427, 2.412, and $6.852 \mathrm{~m}$ in YU1-YU3. In the SM technique, YU1, YU2, and YU3 were $0.532,0.627$, and $0.699 \mathrm{~m}$, respectively; all three values were calculated with an error of less than $1 \mathrm{~m}$. The rate of improvement for YU3 is very high because the low-angle noisy satellites are excluded in the SM technique, and all satellites are included in the WLS method. Consequently, the rate of improvement for YU2 is relatively low since the satellite arrangement is worse than the other points owing to the building's visible range. 
Table 17

Final visible satellites, selected by the signal characteristic classification method, in environment of low-rise building urban.

\begin{tabular}{ccccc}
\hline & & YU1 & & \\
\hline GPS & GLO & QZS & BDS & GAL \\
\hline G10 & & & B6 & \\
G15 & R7 & Q193 & B9 & E19 \\
G20 & & Q194 & B16 & E21 \\
G23 & & B37 & \\
G24 & & YU2 & & \\
\hline & & QZS & BDS & GAL \\
\hline GPS & GLO & & B6 & \\
G10 & & Q193 & B16 & E21 \\
G12 & R7 & Q194 & B19 & \\
G20 & & B37 & \\
G24 & R9 & YU3 & & GAL \\
G32 & & QZS & BDS & \\
\hline GPS & & & B6 & \\
\hline G10 & GLO & B9 & \\
G20 & & B19 & \\
G23 & & Q193 & B36 & \\
G24 & R7 & Q194 & B37 & \\
G25 & R9 & & & \\
G31 & & & & \\
G32 & & & & \\
\hline
\end{tabular}

Table 18

Coordinate calculation value for rate of improvement using SM technique in the environment of low-rise building urban (unit: m).

\begin{tabular}{|c|c|c|c|c|c|c|}
\hline \multicolumn{7}{|c|}{ YU1 } \\
\hline & & & $\Delta X$ & $\Delta Y$ & $\Delta D$ & Rate of improvement \\
\hline VRS & 358874.935 & 177812.522 & & & & \\
\hline WLS & 358877.308 & 177813.031 & 2.373 & 0.509 & 2.427 & \multirow{2}{*}{$356 \%$} \\
\hline SM technique & 358874.492 & 177812.228 & -0.443 & -0.294 & 0.532 & \\
\hline \multicolumn{7}{|c|}{ YU2 } \\
\hline & & & $\Delta X$ & $\Delta Y$ & $\Delta D$ & Rate of improvement \\
\hline VRS & 358819.915 & 177749.554 & & & & \\
\hline WLS & 358820.238 & 177751.944 & 0.323 & 2.390 & 2.412 & \multirow{2}{*}{$285 \%$} \\
\hline SM technique & 358819.485 & 177749.098 & -0.430 & -0.456 & 0.627 & \\
\hline \multicolumn{7}{|c|}{ YU3 } \\
\hline & & & $\Delta X$ & $\Delta Y$ & $\Delta D$ & Rate of improvement \\
\hline VRS & 359117.152 & 177850.339 & & & & \\
\hline WLS & 359123.680 & 177852.420 & 6.528 & 2.081 & 6.852 & \multirow{2}{*}{$880 \%$} \\
\hline SM technique & 359117.670 & 177850.809 & 0.518 & 0.470 & 0.699 & \\
\hline
\end{tabular}

\subsubsection{Environment of high-rise building urban}

In the case of an environment of high-rise building urban, the area surrounding the smartphone measurement location is surrounded by buildings, and the shielding element by the building is a location where the multipath signal by the exterior wall of the building can affect 
precision. In A1, there are five commonly visible satellites analyzed by the signal characteristic classification method. G10, G15, G20, G23, G25, and B26, which were not common satellites in the signal characteristic classification, were selected as final visible satellites because their average correlation coefficients were $0.99,0.98,0.99,0.98,0.98$, and 0.58 , respectively, in the correlation analysis with commonly visible satellites. A2 is an area in which the visible range is opposite to that of A1. A2 is an area where the visible range is formed in the east-west direction, and the multipath signal is considerably generated because the space between buildings, as well as the visible range, is narrow. Ten commonly visible satellites of A2, G12, and G31, which are not common satellites, are excluded from the visible satellites according to the sky mask method. For G20, G23, G25, G32, R7, Q193, B9, and B13, which were not common satellites in the signal characteristic classification, the average correlation coefficients were $0.99,0.98,0.97,0.56,0.78$, $0.01,0.99$, and 0.53 , respectively, in the correlation analysis with commonly visible satellites.

Except for the Q193 satellite, which is below the reference value, the remaining satellites were selected as the final visible satellites. The A3 station has 10 commonly visible satellites, and E8, which is not a common satellite, is excluded from visible satellites according to the sky mask method. G10, G20, G24, G31, B9, B22, B26, and E30, which were not common satellites in the signal characteristic classification, were selected as the final visible satellites because their average correlation coefficients were $0.98,0.40,0.98,0.42,0.99,0.87,0.48$, and 0.99 , respectively, in the correlation analysis with the commonly visible satellites (refer to Table 19).

Table 19

Final selection of visible satellites from optimal combination of visible satellites in environment of high-rise building urban.

\begin{tabular}{|c|c|c|c|c|}
\hline & & A1 & & \\
\hline GPS & GLO & QZS & BDS & GAL \\
\hline G10 & \multirow{6}{*}{ R6 } & \multirow{6}{*}{ - } & & \multirow{6}{*}{$\begin{array}{l}\text { E2 } \\
\text { E8 }\end{array}$} \\
\hline G12 & & & & \\
\hline G15 & & & B16 & \\
\hline G20 & & & B26 & \\
\hline G23 & & & & \\
\hline G25 & & & & \\
\hline \multicolumn{5}{|c|}{$\mathrm{A} 2$} \\
\hline GPS & GLO & QZS & BDS & GAL \\
\hline G10 & & \multirow{6}{*}{ - } & \multirow{6}{*}{$\begin{array}{c}\text { B9 } \\
\text { B13 } \\
\text { B16 } \\
\text { B26 } \\
\text { B36 }\end{array}$} & \multirow{6}{*}{$\begin{array}{l}\text { E2 } \\
\text { E8 }\end{array}$} \\
\hline G20 & R6 & & & \\
\hline G23 & R7 & & & \\
\hline G24 & R9 & & & \\
\hline G25 & \multirow{2}{*}{ R16 } & & & \\
\hline G32 & & & & \\
\hline \multicolumn{5}{|c|}{ A3 } \\
\hline GPS & GLO & QZS & BDS & GAL \\
\hline G10 & \multirow{7}{*}{$\begin{array}{c}\text { R6 } \\
\text { R7 } \\
\text { R16 }\end{array}$} & \multirow{7}{*}{$\begin{array}{l}\text { Q193 } \\
\text { Q194 }\end{array}$} & & \multirow{7}{*}{$\begin{array}{c}\text { E2 } \\
\text { E30 }\end{array}$} \\
\hline G12 & & & B9 & \\
\hline G20 & & & B16 & \\
\hline G24 & & & B22 & \\
\hline G25 & & & B26 & \\
\hline G31 & & & & \\
\hline G32 & & & & \\
\hline
\end{tabular}


The level of shielding by high-rise buildings was higher than in the environment of low-rise building urban, and multiple paths occurred owing to the material of the building surface. Since each study site is located between buildings, it is determined that many abnormal signals due to signal reflection or diffraction are received. It can be seen that the precision of the environment of high-rise building urban is much lower than that in the environment of low-rise building urban owing to such signal noise that affects the coordinate precision. The visible range is narrowed by the buildings' influence, and the effect is due to the poor arrangement of the visible satellites. The distance errors of A1 were $8.551 \mathrm{~m}$ in the WLS method and $6.446 \mathrm{~m}$ in the SM technique, showing an approximately $33 \%$ rate of improvement, and the distance errors of A2 were $18.398 \mathrm{~m}$ in the WLS method and $4.038 \mathrm{~m}$ in the SM technique, showing a $356 \%$ rate of improvement. The distance errors of A3 were $5.840 \mathrm{~m}$ for the WLS method and $5.486 \mathrm{~m}$ for the SM technique, showing an approximately $6 \%$ rate of improvement (Table 20). Overall, A1 and A3 showed some improvement, and A2 showed greater improvement in the $x$-axis direction. Consequently, the multipath of the low-angle satellite signal arranged at right angles to the building near A2 occurred in double or triple, and the signal characteristic value contained great inaccuracy. For this reason, the SM technique was used by excluding the signal characteristic value analysis. Low-angle satellite signals should not be used in areas where there are many buildings and multiple paths, such as in an environment of high-rise building urban, and this error can be reduced by analyzing the signal characteristics of the satellites.

\subsubsection{Environment surrounded by tall buildings}

YU5, an environment surrounded by tall buildings, has a limited visibility range for receiving satellite signals because buildings cover all sides. However, the actual received signal demonstrated more satellite signals than the visible range. Additionally, satellite signals with high to low EAs were received, and low-elevation satellite signals were received through double

Table 20

Coordinate calculation value for rate of improvement using SM technique in the environment of high-rise building urban (unit: m).

\begin{tabular}{|c|c|c|c|c|c|c|}
\hline \multicolumn{7}{|c|}{ A1 } \\
\hline & & & $\Delta X$ & $\Delta Y$ & $\Delta D$ & Rate of improvement \\
\hline $\mathrm{T} / \mathrm{S}$ & 442028.636 & 154713.082 & & & & \\
\hline WLS & 442022.088 & 154707.582 & -6.548 & -5.500 & 8.551 & \multirow{2}{*}{$33 \%$} \\
\hline SM technique & 442026.369 & 154707.048 & -2.267 & -6.034 & 6.446 & \\
\hline \multicolumn{7}{|c|}{ A2 } \\
\hline & & & $\Delta X$ & $\Delta Y$ & $\Delta D$ & Rate of improvement \\
\hline $\mathrm{T} / \mathrm{S}$ & 442044.215 & 154726.119 & & & & \\
\hline WLS & 442062.295 & 154729.525 & 18.080 & 3.406 & 18.398 & \multirow{2}{*}{$356 \%$} \\
\hline SM technique & 442046.936 & 154729.103 & 2.721 & 2.984 & 4.038 & \\
\hline \multicolumn{7}{|c|}{ A3 } \\
\hline & & & $\Delta X$ & $\Delta Y$ & $\Delta D$ & Rate of improvement \\
\hline $\mathrm{T} / \mathrm{S}$ & 442092.075 & 154669.814 & & & & \\
\hline WLS & 442090.265 & 154664.262 & -1.810 & -5.552 & 5.840 & \multirow{2}{*}{$6 \%$} \\
\hline SM technique & 442090.251 & 154664.640 & -1.824 & -5.174 & 5.486 & \\
\hline
\end{tabular}


Table 21

Final selection of visible satellites from the optimal combination of visible satellites in an environment surrounded by tall buildings.

\begin{tabular}{ccccc}
\hline & & YU5 & & \\
\hline GPS & GLO & QZS & BDS & GAL \\
\hline G12 & & & & \\
G25 & R2 & Q193 & B20 & E7 \\
G31 & & Q194 & B37 & E27 \\
G32 & & & & \\
\hline
\end{tabular}

Table 22

Coordinate calculation value for rate of improvement using SM technique in an environment surrounded by tall buildings (unit: $\mathrm{m}$ ).

\begin{tabular}{lllllll}
\hline \multicolumn{7}{c}{ YU5 } \\
\hline T/S & 359617.745 & 177995.668 & $\Delta X$ & $\Delta Y$ & $\Delta D$ & Rate of improvement \\
\hline WLS & 359605.153 & 178000.491 & -12.592 & 4.823 & 13.484 & \multirow{2}{*}{$5 \%$} \\
\cline { 1 - 5 } SM technique & 359605.699 & 178000.015 & -12.046 & 4.347 & 12.806 & \\
\hline
\end{tabular}

and triple multipaths. These signals have a large effect on the position error and cause a reduction in accuracy. YU5 has 9 commonly visible satellites, and R12, R13, B6, B9, B16, and B19, which were not common satellites, are excluded from visible satellites according to the sky mask method. G12, R3, and B20, which were not common satellites in the signal characteristic classification, showed average correlation coefficients of $0.59,0.32$, and 0.89 , respectively, in the correlation analysis with commonly visible satellites. Among them, the R3 satellite below the reference value was excluded, and the remaining G12 and B20 satellites were classified as final visible satellites (Table 21).

The YU5 environment has a narrow visibility range and is surrounded by tall buildings on all sides. Since there are many multipath satellite signals, the accuracy of the position was not significantly improved even when analyzed using the SM technique in this study. The distance errors are $13.484 \mathrm{~m}$ for the WLS method and $12.806 \mathrm{~m}$ for the SM technique, which is an approximately $5 \%$ improvement (Table 22). YU5 has a limited visibility range as buildings surround all directions, and the accuracy degradation rate due to satellite placement is prominent. It is a location that is generally difficult to interpret owing to the severe multipath signal by the buildings on all sides. It is considered that research on satellite signals is necessary for the precision of smartphones in such an environment surrounded by tall buildings.

\section{Conclusions}

In this study, we used the 3D surface model and SM technique to improve the GNSS accuracy of smartphones. To analyze the visibility between a smartphone and a satellite, the GIS analysis and skyline and barrier analysis methods were used to calculate the visible range, the analyzed visible range and altitude of the satellite were calculated, and visible satellites were selected on the basis of the sky mask method. By analyzing the characteristic values of the observed satellites' signal altitude, $\mathrm{C} / \mathrm{No}$, and Prc, visible satellites were classified through the satellite 
signal characteristic analysis. The satellites classified according to the two methods were recombined by statistical analysis to select optimal visible satellites. The location of the smartphone was calculated using the optimal combination of classified satellites, and comparing the calculated location coordinates with the true position coordinates resulted in the following results:

The improvement rate of YU2 is lower than those of YU1 and YU3 in an environment surrounded by buildings, artificial structures, and trees with relatively good reception. The reason is that the arrangement of satellites is worse than those of other points owing to the visible range of buildings. Generally, in the case of the environment of low-rise building urban, the accuracy of the open terrain environment can be secured when the location calculation is performed using the SM technique. In an environment surrounded by buildings with reflective external walls, signal diffraction and multipath occurred, and the improvement in location precision was not high compared with that in the environment surrounded by buildings, artificial structures, and trees. The high rate of improvement for A2 is due to the omission of the signal error from the low-angle satellite. In an environment heavily influenced by buildings, such as an environment of high-rise building urban, the positioning accuracy is improved by omitting the low-angle multipath signal in the analysis of the satellite signal characteristics. Overall, the positional accuracy in the environment of high-rise building urban is about 4 to $6.5 \mathrm{~m}$.

Almost all satellite signals are received through a multipath in an environment surrounded by tall buildings, so it is difficult to calculate the positional accuracy. The distance error of YU5 is improved by about $5 \%$ compared with that using the WLS method. However, the position error with respect to the ground control point is significantly larger than $10 \mathrm{~m}$. The visibility range is limited because the buildings surrounded all sides of the YU5 station, and the accuracy degradation rate due to the satellite arrangement is also significant. Furthermore, the multipath signal is so severe that it is relatively difficult to interpret it by a general analysis method. In this study, the location accuracy of the smartphone GNSS was used by using a 3D surface model and the SM technique that can consider not only the building but also the trees and other structures around the building. After analyzing the receiving satellite signal characteristics, the optimal satellite was selected, and the position was calculated using the selected satellite. Through this study, it is considered that a user's location can be determined within $1 \mathrm{~m}$ in a low-density urban area, giving many ideas for LBS. In the environment of high-rise building urban surrounded by buildings, the visible range is narrow; the diffraction and multipath of the signal occur because of the buildings. Since the satellite arrangement is limited according to the narrow visible range, the error occurs at the same time owing to the satellite signal noise and arrangement, thereby producing a larger error than in the environment of low-rise building urban. In an urban area in such an environment, the precision can be improved when the SM technique, which analyzes the values of the satellites' character signals, is used. In the environment surrounded by tall buildings, the reliability of the positioning accuracy is considerably lowered owing to the double and triple multipaths of the satellite signal. As a future study, it is necessary to develop a filtering technique that reduces the noise of the satellite signal or an algorithm that can more accurately model the multipath error. 


\section{Acknowledgments}

This research was supported by the Basic Science Research Program through the National Research Foundation of Korea (NRF) funded by the Ministry of Education (NRF2020R1I1A3061750) and by an NRF grant funded by the Korean government (MSIT) (No. NRF2021R1A5A8033165).

\section{References}

1 M. Bagheri, M. N. Mladenović, I. Kosonen, and J. K. Nurminen: Sustainability 12 (2020) 5901. https://doi. org/10.3390/su12155901

2 F. An, H. Xu, S. Wen, H. Song, Z. Chen, and W. Guan: Electronics 10 (2021) 1923. https://doi.org/10.3390/ electronics10161923

3 H. Xu: Procedia Eng. 29 (2012) 3454. https://doi.org/10.1016/j.proeng.2012.01.511

4 R. Cheng, W. Hong, J. Wang, and K.W. Lin: Mobile Inf. Syst. 2016 (2016) 1. https://doi.org/10.1155/2016/5032365

5 S. Yeh, W. Hsu, M. Su, C. Chen, and K. Liu: 2009 Int. Conf. Networking, Sensing and Control (2009) 597. https://doi.org/10.1109/ICNSC.2009.4919345

6 A.H. Behzadan, Z. Aziz, C.J. Anumba, and V.R. Kamat: Autom. Constr. 17 (2008) 737. https://doi.org/10.1016/j. autcon.2008.02.002

7 X. Chen, F. Dovis, S. Peng and Y. Morton: IEEE Trans. Aerospace Electron. Syst. 49 (2013) 1555. https://doi. org/10.1109/TAES.2013.6558004

8 S. Malkos: GPS World 27 (2016) 36. https:/www.gpsworld.com/google-to-provide-raw-gnss-measurements/

9 P. D. Groves: 2005 Proc. 18th Int. Technical Meeting of the Satellite Division of the Institute of Navigation (ION GNSS, 2005) 643 .

10 R. Ercek, P. De Doncker, and F. Grenez: 2005 Proc. 18th Int. Technical Meeting of the Satellite Division of the Institute of Navigation (ION GNSS, 2005) 1083.

11 P. D. Groves: J. Navig. 64 (2011) 417. https://doi.org/10.1017/S0373463311000087

12 L. Wang, P. D. Groves, and M.K. Ziebart: 2011 Proc. European Navigation Conf. (ENC, 2011) 11.

13 L. Wang, P. D. Groves, and M. K. Ziebart: J. Inst. Navig. 60 (2013) 195. https://doi.org/10.1002/navi.38

14 L. Wang, P. D. Groves, and M. K. Ziebart: J. Navig. 68 (2015) 411. https://doi.org/10.1017/S0373463314000836

15 P. D. Groves, L. Wang, and M. Ziebart: GPS World 23 (2012) 14. https://www.gpsworld.com/wirelesspersonalnavigationshadow-matching-12550/

16 P. D. Groves, Z. Jiang, L. Wang, and M. Ziebart: 2012 Proc. 6th ESA Workshop on Satellite Navigation Technologies \& European Workshop on GNSS Signals and Signal Processing (Navitec, 2012) 1. https://doi. org/10.1109/NAVITEC.2012.6423047

17 L. Wang, P.D. Groves, and M.K. Ziebart: 2013 Proc. China Satellite Navigation Conf. (CSNC, 2013$) 613$. https://doi.org/10.1007/978-3-642-37407-4_57

18 D. Kim, K. Park, H. Kim, and H. Tae: J. Navig. Port Res. 39 (2015) 7. https://doi.org/10.5394/KINPR.2015.39.1.7

19 T. Suzuki: Adv. Rob. 33 (2019) 602. https://doi.org/10.1080/01691864.2019.1619622

20 H. I. Kim: Improvement of the Real-Time Positioning Accuracy in Urban Canyons through Development of a New Weight Model based on GNSS Signal Strength, PhD Thesis (Department of Geographic Information Engineering, Inha University, Incheon, South Korea, 2015)

21 H. Xu: Intelligent Shadow Matching based on Improved Multi-classifier for Urban Positioning, Master's Thesis (Department of Mechanical Engineering, The Hong Kong Polytechnic University, Hong Kong, China, 2019).

22 R. I. Hartley: Int. J. Comput. Vision 22 (1997) 5. https://doi.org/10.1023/A:1007957826135

23 A. Lucieer, S.M.d. Jong, and D. Turner: Prog. Phys. Geogr. 38 (2014) 97. https://doi.org/10.1177/0309133313515293

24 B. Yang, Q. Li, and W. Shi: Comput. Geosci. 31 (2005) 77. https://doi.org/10.1016/j.cageo.2004.09.011

25 S. Hewitson and J. Wang: GPS Solutions 10 (2006) 155. https://doi.org/10.1007/s10291-005-0016-2

26 A. P. Cerruti, P. M. Kintner, D. E. Gary, L. J. Lanzerotti, E. R. De Paula, and H. B. Vo: Space Weather 4 (2006) S10006. https://doi.org/10.1029/2006SW000254

27 H. Xu, A. Angrisano, S. Gaglione, and L. Hsu: Satell. Navig. 1 (2020) 1. https://doi.org/10.1186/s43020-02000016-W 